\title{
INFINITE DESCENDING CHAINS OF COCOMPACT LATTICES IN KAC-MOODY GROUPS
}

\author{
LISA CARBONE AND LEIGH COBBS
}

\begin{abstract}
Let $A$ be a symmetrizable affine or hyperbolic generalized Cartan matrix. Let $G$ be a locally compact Kac-Moody group associated to $A$ over a finite field $\mathbb{F}_{q}$. We suppose that $G$ has type $\infty$, that is, the Weyl group $W$ of $G$ is a free product of $\mathbb{Z} / 2 \mathbb{Z}$ 's. This includes all locally compact Kac-Moody groups of rank 2 and three possible locally compact rank 3 Kac-Moody groups of noncompact hyperbolic type. For every prime power $q$, we give a sufficient condition for the rank 2 Kac-Moody group $G$ to contain a cocompact lattice $\Gamma \cong M_{q} *_{M_{q} \cap \widetilde{M}_{q}} \widetilde{M}_{q}$ with quotient a simplex, and we show that this condition is satisfied when $q=2^{s}$. If further $M_{q}$ and $\widetilde{M}_{q}$ are abelian, we give a method for constructing an infinite descending chain of cocompact lattices $\ldots \Gamma_{3} \leq \Gamma_{2} \leq \Gamma_{1} \leq \Gamma$. This allows us to characterize each of the quotient graphs of groups $\Gamma_{i} \backslash \backslash X$, the presentations of the $\Gamma_{i}$ and their covolumes, where $X$ is the Tits building of $G$, a homogeneous tree. Our approach is to extend coverings of edge-indexed graphs to covering morphisms of graphs of groups with abelian groupings. This method is not specific to cocompact lattices in Kac-Moody groups and may be used to produce chains of subgroups acting on trees in a general setting. It follows that the lattices constructed in the rank 2 Kac-Moody group have the Haagerup property.

When $\mathrm{q}=2$ and $\operatorname{rank}(G)=3$ we show that $G$ contains a cocompact lattice $\Gamma_{1}^{\prime}$ that acts discretely and cocompactly on a simplicial tree $\mathcal{X}$. The tree $\mathcal{X}$ is naturally embedded in the Tits building $X$ of $G$, a rank 3 hyperbolic building. Moreover $\Gamma_{1}^{\prime} \leq \Lambda^{\prime}$ for a non-discrete subgroup $\Lambda^{\prime} \leq G$ whose quotient $\Lambda^{\prime} \backslash X$ is equal to $G \backslash X$. Using the action of $\Gamma_{1}^{\prime}$ on $\mathcal{X}$ we construct an infinite descending chain of cocompact lattices $\ldots \Gamma_{3}^{\prime} \leq \Gamma_{2}^{\prime} \leq \Gamma_{1}^{\prime}$ in $G$. We also determine the quotient graphs of groups $\Gamma_{i}^{\prime} \backslash \backslash \mathcal{X}$, the presentations of the $\Gamma_{i}^{\prime}$ and their covolumes.
\end{abstract}

\section{INTRODUCTION}

Let $G$ be a completion of Tits' Kac-Moody group functor over a finite field $\mathbb{F}_{q}$. Then $G$ is locally compact and totally disconnected ([CG], $[\mathrm{RR}])$. Completions of the Tits functor have been described by Carbone and Garland ([CG]) and Rémy and Ronan ([RR]). Here we construct cocompact lattices in a certain class of complete Kac-Moody groups, using the Rémy-Ronan completion. If $G$ is the Kac-Moody group of a generalized Cartan matrix $A$, then we call $G$ affine if $A$ is positive semi-definite but not positive definite. If $A$ is neither positive definite nor positive semi-definite, but every proper indecomposable submatrix is either positive definite or positive semi-definite, we say that $G$ has hyperbolic type. If every proper indecomposable submatrix of $A$ is positive definite, we say that $G$ has compact hyperbolic type. Thus if $A$ has a proper indecomposable affine submatrix, we say that $G$ has noncompact hyperbolic type.

We consider here the following class of Kac-Moody groups $G$ over a finite field $\mathbb{F}_{q}$. We suppose that $G$ is either of affine or hyperbolic type, and that $G$ has 'type $\infty$ ', that is, the Weyl group

Date: December 5, 2010.

The first author was supported in part by NSF grant \#DMS-0701176. 
$W$ is a free product of $\mathbb{Z} / 2 \mathbb{Z}$ 's. This coincides with the class of affine or hyperbolic Kac-Moody groups corresponding to generalized Cartan matrices $A=\left(A_{i j}\right)_{i, j \in I}$ where all $m_{i j}$ equal $\infty$ for $i \neq j$. In particular, this includes all rank 2 Kac-Moody groups, whose generalized Cartan matrices form the infinite family

$$
A=\left(\begin{array}{cc}
2 & -a \\
-b & 2
\end{array}\right), a b \in \mathbb{Z}_{\geq 4}
$$

which is of affine type if $a b=4$ and of (compact) hyperbolic type if $a b>4$. 'Symmetrizability' is an important property of the generalized Cartan matrix of a Kac-Moody algebra, necessary for the existence of a well-defined symmetric invariant bilinear form $(\cdot \mid \cdot)$ on the Kac-Moody algebra which plays the role of 'squared length' of a root. The rank 2 generalized Cartan matrices given above are automatically symmetrizable.

If $A$ is of affine type then there are two possible generalized Cartan matrices, namely

$$
A_{1}^{(1)}=\left(\begin{array}{cc}
2 & -2 \\
-2 & 2
\end{array}\right), A_{2}^{(2)}=\left(\begin{array}{cc}
2 & -1 \\
-4 & 2
\end{array}\right) .
$$

For type $A_{1}^{(1)}$, we use a form of the Kac-Moody group such that $G \cong P S L_{2}\left(F_{q}\left(\left(t^{-1}\right)\right)\right)$ (subsection 2.3).

If $\operatorname{rank}(G)=3$, we may apply the classification of symmetrizable hyperbolic Dynkin diagrams ([Sa]) to deduce that $G$ is of noncompact hyperbolic type and that the generalized Cartan matrix of $G$ is one of the following:

$$
A=\left(\begin{array}{ccc}
2 & -2 & -2 \\
-2 & 2 & -2 \\
-2 & -2 & 2
\end{array}\right), A=\left(\begin{array}{ccc}
2 & -4 & -2 \\
-1 & 2 & -1 \\
-2 & -4 & 2
\end{array}\right), A=\left(\begin{array}{ccc}
2 & -1 & -2 \\
-4 & 2 & -4 \\
-2 & -1 & 2
\end{array}\right) \text {. }
$$

In each of these cases $W \cong \mathbb{Z} / 2 \mathbb{Z} * \mathbb{Z} / 2 \mathbb{Z} * \mathbb{Z} / 2 \mathbb{Z}$ and the fundamental chamber for $W$ is an ideal triangle in the hyperbolic plane. Our interest in the Kac-Moody groups in this class comes in part from the fact that the corrected automorphic forms of the corresponding generalized Kac-Moody algebras play an important role in high-energy physics ([GN] and $[\mathrm{HM}]$ ). If $G$ has type $\infty$ and $\operatorname{rank}(G)>3$ then $G$ no longer has hyperbolic type. We will not say more about this case here.

Theorem 1. Let $A$ be a rank 2 affine or hyperbolic generalized Cartan matrix. Let $G$ be a locally compact Kac-Moody group associated to A over a finite field $\mathbb{F}_{q}$. Let $X$ be the Tits building of $G$, the homogeneous tree $X=X_{q+1}$.

(1) For every prime power $q$, there is a finite subgroup $M_{q}(x)$ acting transitively on the edges in the star of a vertex $x \in V X$.

(2) Let $M_{q}=M_{q}\left(x_{1}\right)$ and $\widetilde{M}_{q}=\widetilde{M}_{q}\left(x_{2}\right)$ denote the groups of (1) corresponding to adjacent vertices $x_{1}$ and $x_{2}$ in the Tits building $X$ for $G$. If

$$
\operatorname{Stab}_{M_{q}}^{X}\left(x_{2}\right)=M_{q} \cap \widetilde{M}_{q}=\operatorname{Stab} \frac{X}{M_{q}}\left(x_{1}\right)
$$

then $\Gamma \cong M_{q} *_{M_{q} \cap \widetilde{M}_{q}} \widetilde{M}_{q}$ is a cocompact lattice in $G$ with quotient a simplex.

(3) If further $M_{q}$ and $\widetilde{M}_{q}$ are abelian, there is an infinite descending chain of cocompact lattices $\ldots \Gamma_{3} \leq \Gamma_{2} \leq \Gamma_{1} \leq \Gamma$ with distinct quotient graphs $\Gamma_{i} \backslash X$. 
(4) When $q=2^{s}$, the condition in (2) is satisfied, with $M_{q} \cap \widetilde{M}_{q}=\{1\}$. When $q=2$, the condition in (3) is also satisfied, as $M_{q} \cong \mathbb{Z} / 3 \mathbb{Z} \cong \widetilde{M}_{q}$.

The lattices of Theorem 1 are tree lattices for the homogeneous tree $X=X_{q+1}$. Thus the theories of Bass-Serre and Bass-Lubotzky for constructing lattices via their actions on trees are accessible for the Kac-Moody groups of Theorem 1 ([B], [BL], [L], [S]). While such methods are known to produce ascending and descending chains of subgroups in automorphism groups of trees, our results give the first such embedding of descending chains of lattices in Kac-Moody groups.

We note that the lattice $\mathbb{Z} / 3 \mathbb{Z} * \mathbb{Z} / 3 \mathbb{Z}$ obtained in (4) of Theorem 1 (similarly $\mathbb{Z} / 3 \mathbb{Z} * \mathbb{Z} / 3 \mathbb{Z} * \mathbb{Z} / 3 \mathbb{Z}$ in Theorem 5) is residually finite. In general, by a result of Bass and Kulkarni $([\mathrm{BK}])$, all cocompact tree lattices are finitely generated and virtually free. Thus any cocompact lattice in $G$ has a residually finite subgroup of finite index. It is known that residually finite groups can admit descending chains of subgroups. However, we do not use residual finiteness to construct infinite descending chains over the lattices in Theorem 1. Our strategy is to extend coverings of edge-indexed graphs to covering morphisms of graphs of groups with abelian groupings (Section 3.3). This provides a new tool for constructing descending chains of subgroups in locally compact groups that act on trees. The benefit of this method is that it is constructive and allows us to determine the quotient graphs of groups, the presentations and covolumes of all sublattices in the descending chain. This method is not specific to cocompact lattices in Kac-Moody groups and may be used to produce chains of subgroups acting on trees in a general setting. For example, our method also applies to lattices that are not residually finite, such as nonuniform lattices, though we do not consider this case here.

The sufficient condition stated in (2) of Theorem 1 was first proposed for tree lattices by Lubotzky who proved that this condition is satisfied in $S L_{2}$.

Part (1) of Theorem 1 is adapted from Lemma 3.5 of [L] to the setting of rank 2 affine or hyperbolic Kac-Moody groups using the Levi decomposition of the parabolic subgroups due to [RR] (see below).

Lemma 2. ([L], 3.5) For every prime power $q, S L_{2}\left(\mathbb{F}_{q}\right)$ contains a subgroup $M_{q}$ acting transitively on the projective line $\mathbb{P}^{1}\left(\mathbb{F}_{q}\right)$.

- For $q=11,19,29, A_{5}$ embeds in $P S L_{2}\left(\mathbb{F}_{q}\right)$, so take $M_{q}$ to be the preimage of $A_{5}$ in $S L_{2}\left(\mathbb{F}_{q}\right)$. Then $\left|M_{q}\right|=120$.

- For $q=7, S_{4}$ embeds in $P S L_{2}\left(\mathbb{F}_{q}\right)$, so take $M_{q}$ to be the preimage of $S_{4}$ in $S L_{2}\left(\mathbb{F}_{q}\right)$. Then $\left|M_{q}\right|=48$.

- For $q \notin\left\{2^{s}, 5,7,11,19,29\right\}, M_{q}$ is the normalizer of a non-split Cartan subgroup. Then $\left|M_{q}\right|=2(q+1)$.

- For $q=2^{s}, M_{q}$ is a non-split Cartan subgroup, and hence abelian. Then $\left|M_{q}\right|=q+1$.

- For $q=5, A_{4}$ embeds in $P S L_{2}\left(\mathbb{F}_{q}\right)$, so take $M_{q}$ to be the preimage of $A_{4}$ in $S L_{2}\left(\mathbb{F}_{q}\right)$. Then $\left|M_{q}\right|=24$.

We extend this lemma to the setting of Kac-Moody groups over $\mathbb{F}_{q}$ corresponding to rank 2 affine or hyperbolic generalized Cartan matrices by using the embedding of $[\mathrm{RR}]$ of $S L_{2}\left(\mathbb{F}_{q}\right)$ 
into standard parabolic subgroups of the Kac-Moody group. Rémy and Ronan define the Levi factor of a standard parabolic subgroup of type $i, i=1,2$, as

$$
L_{i}=\left(S L_{2}\left(\mathbb{F}_{q}\right) \times\left(\mathbb{F}_{q}^{\times}\right)\right)_{i}
$$

where $L_{i}$ is the group generated by $H \cong \mathbb{F}_{q}^{\times} \times \mathbb{F}_{q}^{\times}$and the root groups $U_{ \pm \alpha_{i}}$. Rémy and Ronan show that standard parabolic subgroup of type $i$ is the semidirect product

$$
P_{i}=L_{i} \ltimes U^{i},
$$

where $U^{i}$ is a pro- $p$ group and is the normal closure of the group generated by all positive root groups except $U_{\alpha_{i}}$. We refer the reader to $[R R]$ for a definition of the action of the group $L_{i}$ on $U^{i}$. We remark that the Levi factors of parabolic subgroups are isomorphic for rank 2 affine and hyperbolic Kac-Moody groups over $\mathbb{F}_{q}$.

By a slight abuse of notation, we let $M_{q}$ denote the image of the group $M_{q}$ of ([L], Lemma 3.5) in the Levi factor $L_{1}$ and we let $\widetilde{M}_{q}$ denote the image of $M_{q}$ in $L_{2}$. Combining the above results we deduce the following.

Theorem 3. Let $A$ be a rank 2 affine or hyperbolic generalized Cartan matrix. Let $G$ be a locally compact Kac-Moody group associated to A over a finite field $\mathbb{F}_{q}$ in the Rémy-Ronan completion. Let $X=X_{q+1}$ be the Bruhat-Tits tree for $G$. Let $P_{1}$ and $P_{2}$ be the maximal standard parabolic subgroups of $G$. Let $M_{q}$ and $\widetilde{M}_{q}$ be subgroups of $P_{1}$ and $P_{2}$ respectively satisfying

$$
\operatorname{Stab}_{M_{q}}\left(x_{2}\right)=M_{q} \cap \widetilde{M}_{q}=\operatorname{Stab}_{\widetilde{M}_{q}}\left(x_{1}\right)
$$

for adjacent vertices $x_{1}, x_{2} \in V X$. Then $G$ contains a cocompact lattice subgroup $\Gamma=M_{q} *_{M_{q} \cap \widetilde{M}_{q}} \widetilde{M}_{q}$, where

(i) For $q=2^{s}$, we take $M_{q}$ to be the image of the non-split Cartan subgroup of $S L_{2}\left(\mathbb{F}_{q}\right)$ of order $q+1$ in $L_{1}$, and $\widetilde{M}_{q}$ its image in $L_{2}$.

(ii) For $q \notin\left\{2^{s}, 5,7,11,19,29\right\}$, we take $M_{q}$ to be the image of the normalizer of a non-split Cartan subgroup of $S L_{2}\left(\mathbb{F}_{q}\right)$ of order $2(q+1)$ in $L_{1}$, and $\widetilde{M}_{q}$ its image in $L_{2}$.

(iii) For $q=11,19,29, A_{5}$ embeds in $P S L_{2}\left(\mathbb{F}_{q}\right)$, so we take $M_{q}$ to be the preimage of $A_{5}$ in $L_{1}$, and $\widetilde{M}_{q}$ its preimage in $L_{2}$.

(iv) For $q=7, S_{4}$ embeds in $P S L_{2}\left(\mathbb{F}_{q}\right)$, so we take $M_{q}$ to be the preimage of $S_{4}$ in $L_{1}$, and $\widetilde{M}_{q}$ its preimage in $L_{2}$.

(v) For $q=5, A_{4}$ embeds in $P S L_{2}\left(\mathbb{F}_{q}\right)$, so we take $M_{q}$ to be the preimage of $A_{4}$ in $L_{1}$, and $\widetilde{M}_{q}$ its preimage in $L_{2}$.

Theorem 3 assumes the existence of finite subgroups $M_{q}$ and $\widetilde{M}_{q}$ of $P_{1}$ and $P_{2}$ respectively and a sufficient condition on the action of $M_{q}$ and $\widetilde{M}_{q}$ on the Tits building $X$. Using Lemma 2 we deduce the existence of $M_{q} \leq P_{1}$ and $\widetilde{M}_{q} \leq P_{2}$ as in Theorem 3. However, in this work we only verify that $\operatorname{Stab}_{M_{q}}\left(x_{2}\right)=M_{q} \cap \widetilde{M}_{q}=S t a b_{M_{q}}\left(x_{1}\right)$ in case (i) of Theorem 3. We refer the reader to $[\mathrm{CT}]$ where the authors classify all subgroups $M_{q}$ and $\widetilde{M}_{q}$ giving rise to cocompact lattice amalgams in rank 2 locally compact Kac-Moody groups corresponding to symmetric generalized Cartan matrices. 
When $q=2$, we are able to obtain some of the results of Theorem 1 in the rank 3 setting using the following.

Theorem 4. Let $G$ be a locally compact symmetrizable rank 3 Kac-Moody group of type $\infty$ over a finite field $\mathbb{F}_{q}$. Let $X$ be the Tits building of $G$. Then $X$ contains a naturally inscribed bihomogeneous tree $\mathcal{X}=X_{3, q+1}$ which is trivalent when $q=2$.

In [GP] Gaboriau and Paulin considered an ideal hyperbolic triangle of groups with face groups trivial and the edge groups finite groups $A, B$, and $C$. The fundamental group $\Gamma$ of the triangle of groups is the free product $A * B * C$. The authors observe that $\Gamma$ is a discrete subgroup of Aut $(X)$ where $X$ is the universal cover of the triangle of groups, endowed with the compact open topology. By taking the finite groups $A, B$, and $C$ to be copies of $\mathbb{Z} / 3 \mathbb{Z}$ 's, we show that the triangle of groups can be embedded into our rank 3 Kac-Moody groups $G$ of noncompact hyperbolic type, and that its image in $G$ is also discrete (Theorem 5).

Theorem 5. Let $G$ be a symmetrizable locally compact rank 3 Kac-Moody group of type $\infty$ over a finite field $\mathbb{F}_{q}$. Let $X$ be the Tits building of $G$. Suppose that $q=2$.

(1) Let $\Gamma$ denote the free product $\mathbb{Z} / 3 \mathbb{Z} * \mathbb{Z} / 3 \mathbb{Z} * \mathbb{Z} / 3 \mathbb{Z}$. Then $\Gamma$ is an $\mathcal{X}$-lattice for the tree $\mathcal{X}$ of Theorem 4 .

(2) The image $\Gamma_{1}^{\prime}$ of $\Gamma$ in $G$ is a cocompact lattice in $G$.

(3) There is non-discrete subgroup $\Lambda^{\prime} \leq G$ with $\Gamma_{1}^{\prime} \leq \Lambda^{\prime}$ and $\Lambda^{\prime} \backslash X$ a simplex (ideal triangle) in the Tits building $X$.

(4) There is an infinite descending chain $\ldots \Gamma_{3}^{\prime} \leq \Gamma_{2}^{\prime} \leq \Gamma_{1}^{\prime}$ of cocompact lattices in $G$.

To produce the infinite descending chain of lattices in (4) of Theorem 5 we use the action of $\Gamma_{1}^{\prime}$ on the simplicial tree $\mathcal{X}$. The group $G$ acts on $\mathcal{X}$ with quotient a tripod. The subgroup $\Gamma_{1}^{\prime} \leq G$ acts cocompactly with finite stabilizers. Moreover $\Gamma_{1}^{\prime}$ is isomorphic to the group $\Gamma_{1}$ in the infinite descending chain of Theorem 1 in the rank 2 Kac-Moody group over $\mathbb{F}_{2}$. Thus the construction of the rank 2 chain also gives the construction of the chain (4) in the rank 3 setting. We construct the resulting quotient graphs of groups $\Gamma_{i}^{\prime} \backslash \backslash \mathcal{X}$ for the lattices $\Gamma_{i}^{\prime}$, we give the presentations of the $\Gamma_{i}^{\prime}$ and we compute their covolumes. Using a covering morphism of graphs of groups we embed $\Gamma$ into $G$. We construct a covering morphism of triangles of groups to exhibit the nondiscrete subgroup $\Lambda^{\prime} \leq G$ in Theorem 1 (Section 5).

When $G$ has rank 3 , the tree $\mathcal{X}$ inscribed in the Tits building $X$ of $G$ is homogeneous only when $q=2$. Since our results depend on the homogeneity of $\mathcal{X}$, we do not expect our methods in rank 3 to extend easily to other values of $q$, though a generalization may be possible. Our methods for rank 3 groups might also extend beyond the class of Kac-Moody groups of type $\infty$, however, this class of groups is most compatible with our constructions in rank 2.

The existence of cocompact lattices in certain Kac-Moody groups has already been established by Carbone and Garland ([CG]) and by Rémy and Ronan ([RR]). In $[\mathrm{CG}]$, Carbone and Garland generalized Lubotzky's construction of Schottky groups of automorphisms in $S L_{2}$ over a nonarchimedean local field to give torsion free cocompact lattices in any rank 2 locally compact Kac-Moody group over a finite field $\mathbb{F}_{q}$. In $[\mathrm{CT}]$ the authors classified cocompact lattices with torsion and with quotient a simplex in rank 2 Kac-Moody groups corresponding to symmetric generalized Cartan matrices. Their classification includes our examples for $q=2^{s}$ of Theorem 1 when the generalized Cartan matrix is symmetric, though our construction of descending chains of cocompact lattices holds for a general $2 \times 2$ generalized Cartan matrix. 
In [Bo1] and [Bo2], Bourdon constructed a family of cocompact lattices in the automorphism groups of certain hyperbolic Kac-Moody buildings. Here 'hyperbolic' means that the Coxeter group comes from a tiling of some hyperbolic space, but the fundamental domain is not required to be a simplex. In [RR], Rémy and Ronan showed that Bourdon's cocompact lattices $\Gamma_{r, q+1}, r \geq 5, q \geq 3$, can be embedded into the closure of right-angled Kac-Moody groups in the automorphism groups of their buildings, $I_{r, q+1}$ for $q$ a prime power. The buildings $I_{r, q+1}$ are right-angled Fuchsian buildings whose associated Weyl groups are the hyperbolic reflection groups arising from a tiling of the hyperbolic plane by regular right-angled $r$-gons (though the fundamental domain is not necessarily a simplex). The link at each vertex of $I_{r, q+1}$ is the complete bipartite graph of type $(q+1, q+1)$ (see also [Re]). The images of the lattices $\Gamma_{r, q+1}$ in the Kac-Moody group are given by the presentations

$$
\Gamma_{r, q+1}=\left\langle\left\{\gamma_{i}\right\}_{i \in \mathbb{Z} / r \mathbb{Z}} \mid \gamma_{i}^{q+1}=1,\left[\gamma_{i}, \gamma_{i+1}\right]=1\right\rangle .
$$

The Kac-Moody groups of type $\infty$ we consider here are examples of right-angled Kac-Moody groups, however there is no overlap with the setting of $[R R]$. The analog of ' $r$ ' in our results, that is, the type of fundamental polygon for the Weyl group, is 2 or 3 , while the results of [RR] and [Bo1], [Bo2] hold only for $r \geq 5$. It is intriguing however that the lattices of [RR] (Section 5C) appear to be quotients of the lattices we construct in the rank 3 Kac-Moody group.

Using actions of Kac-Moody groups on their Tits buildings, in [C1] the author showed that all symmetrizable affine or hyperbolic locally compact Kac-Moody groups $G$ of either rank 2 or of rank 3 noncompact hyperbolic type have the Haagerup property. Since the Haagerup property for a locally compact group $G$ implies the Haagerup property for a lattice subgroup $\Gamma \leq G$, the lattices of the descending chains of Theorems 1 and 5 have the Haagerup property.

The authors are grateful to Frédéric Paulin for explaining his construction to us and to Delaram Kahrobaei for helpful discussions. We thank Bertrand Rémy for clarifying the details of his paper with Ronan ([RR]). We are indebted to Gabriel Rosenberg for teaching us how to extend coverings of edge-indexed graphs to covering morphisms of graphs of groups (Section 3.3). Some of our results were obtained independently by Inna Capdeboscq (Korchagina) and Anne Thomas who gave a detailed classification of lattices in rank 2 locally compact Kac-Moody groups with quotient a simplex. We refer the reader to their informative paper [CT] and we thank them for helpful discussions during the early phase of this work. We also thank Anne Thomas for explaining to us how to construct the covering morphism of Theorem 1 for rank 3 Kac-Moody groups. We are grateful to the referee for suggestions that improved a number of our statements. We also thank the editor for helpful comments.

\section{LOCALly COMPACT KAC-MOOdY GROUPS}

Though there is no obvious infinite dimensional generalization of finite dimensional Lie groups, Tits associated a group functor $G_{A}$ on the category of commutative rings, such that for any symmetrizable generalized Cartan matrix $A$ and any $\operatorname{ring} R$ there exists a group $G_{A}(R)$ ([Ti1], [Ti2]). Tits defined not one group, but rather minimal and maximal groups. The value of the Tits functor $G_{A}$ over a field $k$ is called a minimal Kac-Moody group. The maximal or complete Kac-Moody group is defined relative to a completion of the Kac-Moody algebra and contains $G_{A}(k)$ as a dense subgroup. 
2.1. Tits' presentation of minimal Kac-Moody groups. In this subsection we define minimal Kac-Moody groups over arbitrary fields by generators and relations, following Tits ([Ti1]).

Let $\mathfrak{g}$ be a symmetrizable Kac-Moody algebra with Cartan subalgebra $\mathfrak{h}$. For each simple root $\alpha_{i}, i \in I=\{1, \ldots \ell\}$, we define the simple root reflection

$$
w_{i}\left(\alpha_{j}\right):=\alpha_{j}-\alpha_{j}\left(\alpha_{i}^{\vee}\right) \alpha_{i} .
$$

The $w_{i}$ generate a subgroup $W=W(A) \subseteq A u t\left(\mathfrak{h}^{*}\right)$, called the Weyl group of $A$. We introduce an auxiliary group $W^{*} \subseteq A u t(\mathfrak{g})$, generated by elements $\left\{w_{i}^{*}\right\}_{i \in I}$, where

$$
w_{i}^{*}=\exp \left(\operatorname{ad}\left(e_{i}\right)\right) \exp \left(-a d\left(f_{i}\right)\right) \exp \left(a d\left(e_{i}\right)\right)=\exp \left(-\operatorname{ad}\left(f_{i}\right)\right) \exp \left(\operatorname{ad}\left(e_{i}\right)\right) \exp \left(-\operatorname{ad}\left(f_{i}\right)\right) .
$$

There is a surjective homomorphism $\epsilon: W^{*} \rightarrow W$ which sends $w_{i}^{*}$ to $w_{i}$ for all $i$. We define certain elements of $\mathfrak{g}$, denoted $\left\{e_{\alpha}\right\}_{\alpha \in \Phi}$, where $\Phi$ denotes the set of real roots of $\mathfrak{g}$. Given $\alpha \in \Phi$, write $\alpha$ in the form $w \alpha_{j}$ for some $j \in I$ and $w \in W$, choose $w^{*} \in W^{*}$ which maps onto $w$, and set $e_{\alpha}=w^{*} e_{\alpha_{j}}$. It is clear from [Ti1, (3.3.2)] that $e_{\alpha}$ belongs to the root space $g^{\alpha}, e_{\alpha}$ is uniquely determined up to sign, and for all $i \in I, w_{i}^{*} e_{\alpha}=\eta_{\alpha, i} e_{w_{i} \alpha}$ for some constants $\eta_{\alpha, i} \in\{ \pm 1\}$.

Let $A$ be a symmetrizable generalized Cartan matrix. Let $k$ denote an arbitrary field. The group $G=G_{A}(k)$ defined below is called the incomplete simply-connected Kac-Moody group corresponding to $A$.

By definition, $G_{A}(k)$ is generated by the set of symbols $\left\{\chi_{\alpha}(u) \mid \alpha \in \Phi, u \in k\right\}$ satisfying relations (R1)-(R7) below. In all the relations $i, j$ are elements of $I, u, v$ are elements of $k$ and $\alpha$ and $\beta$ are real roots.

(R1) $\chi_{\alpha}(u+v)=\chi_{\alpha}(u) \chi_{\alpha}(v)$;

(R2) Let $(\alpha, \beta)$ be a prenilpotent pair, that is, there exist $w, w^{\prime} \in W$ such that

$$
w \alpha, w \beta \in \Phi^{+} \text {and } w^{\prime} \alpha, w^{\prime} \beta \in \Phi^{-} .
$$

Then

$$
\left[\chi_{\alpha}(u), \chi_{\beta}(v)\right]=\prod_{m, n \geq 1} \chi_{m \alpha+n \beta}\left(C_{m n \alpha \beta} u^{m} v^{n}\right)
$$

where the product on the right-hand side is taken over all real roots of the form $m \alpha+n \beta$, $m, n \geq 1$, in some fixed order, and $C_{m n \alpha \beta}$ are integers independent of $k$ (but depending on the order). This product appearing on the right-hand side is finite.

For each $i \in I$ set

$$
\begin{aligned}
& \chi_{ \pm i}(u)=\chi_{ \pm \alpha_{i}}(u), u \in k \\
& \widetilde{w}_{i}(u)=\chi_{i}(u) \chi_{-i}\left(-u^{-1}\right) \chi_{i}(u), u \in k \\
& \widetilde{w}_{i}=\widetilde{w}_{i}(1) \text { and } h_{i}(u)=\widetilde{w}_{i}(u) \widetilde{w}_{i}^{-1}, u \in k^{*} .
\end{aligned}
$$

The remaining relations are

(R3) $\widetilde{w}_{i} \chi_{\alpha}(u) \widetilde{w}_{i}^{-1}=\chi_{w_{i} \alpha}\left(\eta_{\alpha, i} u\right)$,

(R4) $h_{i}(u) \chi_{\alpha}(v) h_{i}(u)^{-1}=\chi_{\alpha}\left(v u^{\left\langle\alpha, \alpha_{i}^{\vee}\right\rangle}\right)$ for $u \in k^{*}$,

(R5) $\widetilde{w}_{i} h_{j}(u) \widetilde{w}_{i}^{-1}=h_{j}(u) h_{i}\left(u^{-a_{j i}}\right)$,

(R6) $h_{i}(u v)=h_{i}(u) h_{i}(v)$ for $u, v \in k^{*}$, and

(R7) $\left[h_{i}(u), h_{j}(v)\right]=1$ for $u, v \in k^{*}$.

An immediate consequence of relations (R3) is that $G_{A}(k)$ is generated by $\left\{\chi_{ \pm i}(u)\right\}$. The elements $\widetilde{w}_{i}$ generate a group $\widetilde{W}$ which is isomorphic to the group $W^{*}$ above. 
2.2. BN-pair and Tits building of a minimal Kac-Moody group. Tits' Kac-Moody group functor may be described by certain group theoretic data, called a Tits system or $(B, N)$-pair. This data determines a simplicial complex $X$ called the Tits building on which the group acts. Here we describe briefly the $(B, N)$-pair associated to minimal Kac-Moody group (Tits' functor) over a finite field.

A minimal Kac-Moody group $G$ (Tits' functor) over the finite field $\mathbb{F}_{q}$ has subgroups $B^{ \pm} \subseteq G$, $N \subseteq G$, and Weyl group $W=N / H$, where $H=N \cap B^{ \pm}$is a normal subgroup of $N$. There is a surjective homomorphism $\nu: N \longrightarrow W$. We identify $W$ (non-canonically) with a subset (not a subgroup) of $N$ which contains exactly one representative of every element of $W$. By abuse of notation, this set of representatives will also be called $W$. This coincides with the Weyl group $W$ of the previous section. We have $B^{ \pm}=H U^{ \pm}$where $U^{+}$is generated by all positive real root groups and $U^{-}$is generated by all negative real root groups. Then $\left(B^{+}, N\right)$ and $\left(B^{-}, N\right)$ are $B N$-pairs, and

It follows that

$$
G=B^{+} N B^{-}=B^{-} N B^{+} \text {. }
$$

$$
G=\sqcup_{w \in W} B^{ \pm} w B^{ \pm}
$$

Let $S$ be the standard generating set for the Weyl group $W$ consisting of simple root reflections. Let $U \subsetneq S$. The standard parabolic subgroups are

$$
P_{U}=\sqcup_{w \in\langle U\rangle} B^{ \pm} w B^{ \pm}
$$

A parabolic subgroup is any subgroup containing a conjugate of $B^{ \pm}$. The Tits building of $G$ is a simplicial complex $X$ of dimension $\operatorname{dim}(X)=|S|-1$. In fact we associate a building $X^{ \pm}$ to each $B N$-pair $\left(B^{+}, N\right)$ and $\left(B^{-}, N\right)$. The buildings $X^{+}$and $X^{-}$are isomorphic as chamber complexes and have constant thickness $q+1$ (see [DJ], Appendix KMT).

The vertices of $X$ are given by cosets of the maximal parabolic subgroups in $G$. The incidence relation is described as follows. The $r+1$ vertices $P_{1}, \ldots, P_{r+1}$ span an $r$-simplex if and only if the intersection $P_{1} \cap \cdots \cap P_{r+1}$ is parabolic, that is, contains a conjugate of $B^{ \pm}$. If the root system is infinite, the Weyl group $W$ is infinite, so by the Solomon-Tits theorem, $X$ is contractible. The group $G$ acts by left multiplication on cosets.

2.3. Complete Kac-Moody groups. Let $A$ be an $\ell \times \ell$ symmetrizable generalized Cartan matrix. Let $\widehat{G}=G_{A}\left(\mathbb{F}_{q}\right)$ be a completion of Tits' functor associated to $A$ and the finite field $\mathbb{F}_{q}$. The existence of such a completion was noted by Tits ([Ti2]). Explicit completions have been constructed using distinct methods by Carbone and Garland ([CG]) and by Rémy and Ronan $([\mathrm{RR}])$. A complete Kac-Moody group $\widehat{G}$ over a finite field is locally compact, totally disconnected and the Tits building $X$ is locally finite. We shall primarily use the Rémy-Ronan completion, which we review in this subsection. From now on, we write $B=B^{+}$and $U=U^{+}$.

Let $X$ be the building associated with the positive BN-pair $(B, N)$, and consider the action of $G$ on $X$. We define a topology on $G$ by taking a subbase of neighborhoods of the identity to consist of stabilizers of vertices of $X$. We shall call this topology the building topology. The completion of $G$ in its building topology will be referred to as the Rémy-Ronan completion and denoted by $\widehat{G}$. Let $Z$ be the kernel of the natural map $G \rightarrow \widehat{G}$ (or, equivalently, the kernel of the action of $G$ on $X$ ). Using results of Kac and Peterson [KP], Rémy and Ronan [RR, 1.B] showed that $Z$ is a subgroup of $H$ and hence is finite. Furthermore, $Z$ coincides with the center of $G$. 
Now let $\widehat{B}$ (resp. $\widehat{U}$ ) be the closure of $B$ (respectively $U$ ) in $\widehat{G}$. The natural images of $N$ and $H$ in $\widehat{G}$ are discrete, and therefore we will denote them by the same symbols (without hats).

The following theorem is a collection of results from $[\mathrm{Re}]$ and $[\mathrm{RR}]$ :

Theorem 6. Let $\widehat{G}, \widehat{B}$ and $N$ be as above. Then:

(a) The pair $(\widehat{B}, N)$ is a BN-pair of $\widehat{G}$. Moreover, if $\widehat{X}^{+}$is the associated building, there exists a $\widehat{G}$-equivariant isomorphism between $X^{+}$and $\widehat{X}^{+}$. In particular, the Coxeter group associated to $(\widehat{B}, N)$ is isomorphic to $W=W(A)$.

(b) The group $\widehat{B}$ is an open profinite subgroup of $\widehat{G}$. Furthermore, $\widehat{U}$ is an open pro-p subgroup of $\widehat{B}$.

As is shown in [CER], the Rémy-Ronan completion of the Kac-Moody group $G$ of type $A_{1}^{(1)}$ over $\mathbb{F}$ is isomorphic to $P S L_{2}\left(\mathbb{F}_{q}\left(\left(t^{-1}\right)\right)\right)$.

In the remainder of the paper, we will assume that our Kac-Moody group $G$ is Rémy-Ronan complete, and we drop the 'hats' in the description of the Kac-Moody group $G$.

2.4. Kac-Moody Groups of Type $\infty$. Let $G$ be a Kac-Moody group and suppose that $G$ has type $\infty$. Then the associated Weyl group $W=N / H$, as described using the $B N$-pair, is a free product of copies of $\mathbb{Z} / 2 \mathbb{Z}$. In particular, let $G$ be a Kac-Moody group of type $\infty$ over a finite field $\mathbb{F}_{q}$. Then the Weyl group has the form

$$
W=\left\langle w_{i} \mid i=1, \ldots, n\right\rangle \cong *_{i=1, \ldots, n} \mathbb{Z} / 2 \mathbb{Z},
$$

where $n=\operatorname{rank}(G)$. If $G$ is affine or hyperbolic and has type $\infty$, then $G$ has rank 2 or rank 3 .

\section{Rank 2}

Let $G$ be a locally compact rank 2 affine or hyperbolic Kac-Moody group over $\mathbb{F}_{q}$. Then

$$
W=\mathbb{Z} / 2 \mathbb{Z} * \mathbb{Z} / 2 \mathbb{Z} .
$$

The Tits building $X$ is the $(q+1)$-regular tree $X_{q+1}$. The vertex set $V X$ is given by the set of cosets of the maximal standard parabolic subgroups

$$
\begin{gathered}
P_{1}:=\quad B \sqcup B w_{1} B, \text { and } \\
P_{2}:=\quad B \sqcup B w_{2} B .
\end{gathered}
$$

The oriented edge set is given by

$$
E X=G / B \sqcup \overline{G / B},
$$

where $\overline{G / B}$ denotes the edges of opposite orientation.

The tree $X$ is a homogeneous, bipartite tree (see Figure 1)of degree

$$
\left[P_{1}: B\right]=\left[P_{2}: B\right]=q+1 \text {. }
$$

Since $G$ acts with two orbits on vertices and a single orbit on edges, we can recover, by application of the fundamental theorem of Bass-Serre [S], a presentation for $G$ as an amalgamated free product

$$
G=P_{1} *_{B} P_{2} .
$$

\section{Rank 3}




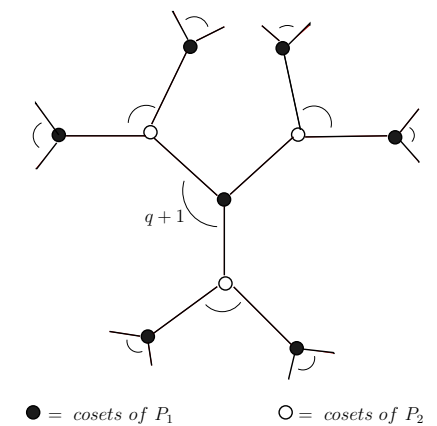

Figure 1. Bruhat-Tits tree for rank 2 Kac-Moody group over $\mathbb{F}_{q}$

Let $G$ be a symmetrizable locally compact rank 3 Kac-Moody group of type $\infty$ over a finite field $\mathbb{F}_{q}$. Then

$$
W=\mathbb{Z} / 2 \mathbb{Z} * \mathbb{Z} / 2 \mathbb{Z} * \mathbb{Z} / 2 \mathbb{Z} .
$$

As we mentioned in the introduction, we may apply the classification of symmetrizable hyperbolic Dynkin diagrams ([Sa]) to deduce that $G$ is of noncompact hyperbolic type and that the generalized Cartan matrix of $G$ is one of the following:

$$
A=\left(\begin{array}{ccc}
2 & -2 & -2 \\
-2 & 2 & -1 \\
-2 & -1 & 2
\end{array}\right), A=\left(\begin{array}{ccc}
2 & -4 & -2 \\
-1 & 2 & -1 \\
-2 & -1 & 2
\end{array}\right), A=\left(\begin{array}{ccc}
2 & -1 & -2 \\
-4 & 2 & -1 \\
-2 & -1 & 2
\end{array}\right) \text {. }
$$

The Tits building $X$ consists of the hyperbolic plane tessellated by ideal triangles, together with $q-1$ additional triangles glued along each edge of a triangle in the hyperbolic plane. The vertices $V X$ of ideal triangles are given by the set of cosets of the maximal standard parabolic subgroups

$$
\begin{aligned}
P_{1,2} & :=\bigsqcup_{w \in<w_{1}, w_{2}>} B w B \text {, and } \\
P_{2,3} & :=\bigsqcup_{w \in<w_{2}, w_{3}>} B w B \text {, and } \\
P_{1,3} & :=\bigsqcup_{w \in<w_{1}, w_{3}>} B w B .
\end{aligned}
$$

The edges correspond to cosets of

$$
\begin{gathered}
Q_{1}:=B \sqcup B w_{1} B, \text { and } \\
Q_{2}:=B \sqcup B w_{2} B, \text { and } \\
Q_{3}:=\quad B \sqcup B w_{3} B .
\end{gathered}
$$

The triangular faces correspond to cosets of $\mathrm{B}$.

We can embed a $(q+1,3)$-bihomogeneous tree in $X$ by taking the maximal tree $\mathcal{X}$ of the barycentric subdivision, as shown in Figure 2. The action of $G$ on $X$ induces an action on $\mathcal{X}$.

For a Kac-Moody group of type $\infty$ over a field of two elements, we will use the action of $G$ on its building (or embedded tree) to exhibit lattice subgroups by constructing their quotient graphs of groups. This technique is explained in the next section. 


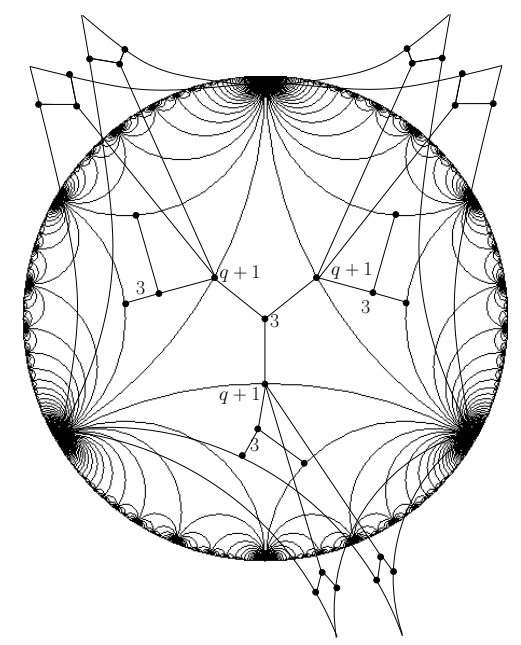

Figure 2. Tits building for a rank 3 Kac-Moody group with $(3, q+1)$ bihomogeneous inscribed tree

\section{LATTICES AND COVERING THEORY}

Let $G$ be a locally compact group acting on a set $X$ with compact open stabilizers, and let $\mu$ be a (left) Haar measure on $G$. Let $\Gamma \leq G$ be a discrete subgroup with quotient $p: G \longrightarrow \Gamma \backslash G$. We call $\Gamma$ a $G$-lattice if $\mu(\Gamma \backslash G)<\infty$, and a uniform or cocompact $G$-lattice if $\Gamma \backslash G$ is compact. When $G$ is unimodular, $\mu\left(G_{x}\right)$ is constant on $G$-orbits, so we can define:

$$
\mu(G \backslash \backslash X):=\sum_{x \in V(G \backslash X)} \frac{1}{\mu\left(G_{x}\right)},
$$

where $G_{x}$ denotes the stabilizer in $G$ of the vertex $x$ and $V(G \backslash X)$ denotes the set of vertices of the quotient graph $G \backslash X$.

Theorem 7. ([BL], (1.6)) Suppose that a group $G$ acts on a set $X$ with compact open stabilizers. For a discrete subgroup $\Gamma \leq G$, the following conditions are equivalent:

(a) $\operatorname{Vol}(\Gamma \backslash \backslash X)<\infty$.

(b) $\Gamma$ is a $G$-lattice (hence $G$ is unimodular), and $\mu(G \backslash \backslash X)<\infty$.

In this case:

$$
\operatorname{Vol}(\Gamma \backslash \backslash X)=\mu(\Gamma \backslash G) \cdot \mu(G \backslash \backslash X)
$$

Let $\Gamma \leq G$ be discrete. Then the diagram of natural projections

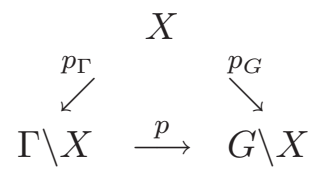

commutes. Assume that $\operatorname{Vol}(\Gamma \backslash \backslash X)<\infty$. Then $\Gamma$ is a $G$-lattice. To determine if $\Gamma$ is uniform or non-uniform in $G$, we use the following:

Lemma 8. ([BL], (1.5.8)) Let $X$ be a set with compact open stabilizers and let $x \in X$. The following conditions are equivalent: 
(a) $\Gamma$ is a uniform $G$-lattice.

(b) Some fiber $p^{-1}\left(p_{G}(x)\right) \cong \Gamma \backslash G / G_{x}$ is finite.

(c) Every fiber of $p$ is finite.

Now let $G$ be a Kac-Moody group of noncompact hyperbolic type. Let $X$ be the Tits building of $G$. Then $G \backslash X$ is not compact. Suppose that $G$ contains a cocompact $G$-lattice $\Gamma$. By the Lemma above, this implies that $\Gamma \backslash G / G_{x}$ is finite for any $x \in V X$, that is, $\Gamma \backslash G / g P_{i} g^{-1}$ is finite for any $g \in G$, where $P_{i}$ is a maximal parabolic subgroup of $G$. Even though $G \backslash X$ is not compact, the number of orbits of $G$ on $X$ is finite.

3.1. Edge-indexed graphs. Let $A$ be a connected locally finite graph, with sets $V A$ of vertices and $E A$ of oriented edges. The initial and terminal vertices of $e \in E A$ are denoted by $\partial_{0} e$ and $\partial_{1} e$ respectively. The map $e \mapsto \bar{e}$ is orientation reversal, with $\partial_{1-j} \bar{e}=\partial_{j}$ e for $j=0,1$.

A graph of groups $\mathbb{A}=\left(A, \mathcal{A}_{v}, \mathcal{A}_{e}, \alpha_{e}\right)$ over a connected graph $A$ consists of an assignment of vertex groups $\mathcal{A}_{v}$ for each $v \in V A$ and edge groups $\mathcal{A}_{e}=\mathcal{A}_{\bar{e}}$ for each $e \in E A$, together with monomorphisms $\alpha_{e}: \mathcal{A}_{e} \rightarrow \mathcal{A}_{\partial_{0} e}$ for each $e \in E A$. We refer the reader to [B] for the definitions of the fundamental group $\pi_{1}\left(\mathbb{A}, a_{0}\right)$ and universal covering tree $\left.X=\widetilde{\left(A, a_{0}\right.}\right)$ of a graph of groups $\mathbb{A}=\left(A, \mathcal{A}_{v}, \mathcal{A}_{e}, \alpha_{e}\right)$, with respect to a basepoint $a_{0} \in V A$.

An edge-indexed graph $(A, i)$ consists of an underlying graph $A$ together with an assignment of a positive integer $i(e) \in \mathbb{Z}_{>0}$ to each edge $e \in E A$. Let $\mathbb{A}=(A, \mathcal{A})$ be a graph of groups. Then $\mathbb{A}$ naturally gives rise to an edge-indexed graph $I(\mathbb{A})=(A, i)$, with for each $e \in E A$, the map $i: E A \rightarrow \mathbb{Z}_{>0}$ given by $i(e)=\left[\mathcal{A}_{\partial_{0} e}: \alpha_{e} \mathcal{A}_{e}\right]$, which we assume to be finite.

Given an edge-indexed graph $(A, i)$, a graph of groups $\mathbb{A}$ such that $I(\mathbb{A})=(A, i)$ is called a grouping of $(A, i)$. We call $\mathbb{A}$ a finite grouping if the vertex groups $\mathcal{A}_{a}$ are all finite, and a faithful grouping if $\mathbb{A}$ is a faithful graph of groups, that is if the fundamental group $\pi_{1}\left(\mathbb{A}, a_{0}\right)$ acts faithfully on the universal covering tree $\left.X=\widetilde{\left(\mathbb{A}, a_{0}\right.}\right)$.

As an example, let $G$ be a rank 2 Kac-Moody group over a finite field $\mathbb{F}_{q}$. The action of $G$ on $X=X_{q+1}$ gives rise to a quotient graph of groups, whose vertices and edges are the $G$-orbits of vertices and edges in $X$.

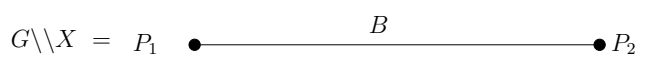

FiguRE 3. graph of groups for a rank 2 Kac-Moody group

The Kac-Moody group $G$ is the fundamental group of this graph of groups, and the Tits building $X$ is the universal covering tree. The corresponding edge-indexed graph is a pair of edges $\{e, \bar{e}\}$ with $i(e)=i(\bar{e})=\left[P_{i}: B\right]=q+1$.

We now describe a method for constructing lattices in $\operatorname{Aut}(X)$ which follows naturally from the fundamental theory of Bass-Serre (see $[\mathrm{B}],[\mathrm{S}]$ ), and was first suggested in $[\mathrm{BK}]$. We begin with an edge-indexed graph $(A, i)$. Then $(A, i)$ determines a universal covering tree $X=\left(\widetilde{A, i, a_{0}}\right)$ up to isomorphism. Let $\mathbb{A}$ be a finite grouping of $(A, i)$. Then there is a homomorphism

$$
\pi_{1}\left(\mathbb{A}, a_{0}\right) \rightarrow \operatorname{Aut}(X) .
$$


This map is a monomorphism if and only if $\mathbb{A}$ is faithful, in which case we may identify $\pi_{1}\left(\mathbb{A}, a_{0}\right)$ with its image in $\operatorname{Aut}(X)$. Since $\mathbb{A}$ is a finite grouping, this image is discrete. By the discussion in Section 3.1 above, the image of $\pi_{1}\left(\mathbb{A}, a_{0}\right)$ is a lattice in $\operatorname{Aut}(X)$ if and only if $\mathbb{A}$ is a faithful graph of finite groups of finite volume.

3.2. Coverings. We have described in Section 3.1 above how to construct lattices in $\operatorname{Aut}(X)$ as fundamental groups $\Gamma$ of graphs of groups. In order to determine if such a $\Gamma$ embeds into a subgroup $G<\operatorname{Aut}(X)$ such as a Kac-Moody group, we will use covering morphisms of graphs of groups.

Definition 1. Let $\mathbb{A}=\left(A, \mathcal{A}_{v}, \mathcal{A}_{e}, \alpha_{e}\right)$ and $\mathbb{A}^{\prime}=\left(A^{\prime}, \mathcal{A}_{v}^{\prime}, \mathcal{A}_{e}^{\prime}, \alpha_{e}^{\prime}\right)$ be graphs of groups. $A$ covering morphism $\Phi=(\varphi,(\delta)): \mathbb{A} \rightarrow \mathbb{A}^{\prime}$ consists of:

(1) a graph morphism $\varphi: A \rightarrow A^{\prime}$;

(2) monomorphisms

$$
\varphi_{a}: \mathcal{A}_{a} \rightarrow \mathcal{A}_{\varphi(a)}^{\prime} \quad(a \in V A), \quad \varphi_{e}=\varphi_{\bar{e}}: \mathcal{A}_{e} \rightarrow \mathcal{A}_{\varphi(e)}^{\prime} \quad(e \in E A)
$$

(3) For each $e \in E A$ with $a=\partial_{0} e$ an element $\delta_{e} \in \mathcal{A}_{\varphi(a)}^{\prime}$ such that the following two conditions hold:

(a) The following diagram commutes:

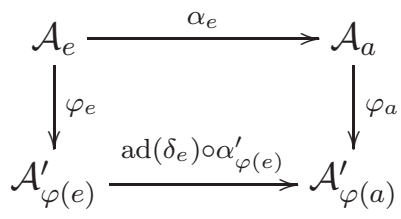

where $\operatorname{ad}(x)(s)=x s x^{-1}$.

(b) For $f \in E A^{\prime}, a^{\prime}=\partial_{0} f$ and $a \in \varphi^{-1}\left(a^{\prime}\right)$, the map

$$
\Phi_{a / f}:\left(\coprod_{e \in \varphi_{(a)}^{-1}(f)} \mathcal{A}_{a} / \alpha_{e} \mathcal{A}_{e}\right) \longrightarrow \mathcal{A}_{\varphi(a)}^{\prime} / \alpha_{f}^{\prime} \mathcal{A}_{f}^{\prime}
$$

defined by

$$
\Phi_{a / f}\left([s]_{e}\right)=\left[\varphi_{a}(s) \delta_{e}\right]_{f}
$$

is bijective (where $s \in \mathcal{A}_{a},[s]_{e}$ is the class of $s$ in $\mathcal{A}_{a} / \alpha_{e} \mathcal{A}_{e}$, and $\varphi_{(a)}^{-1}(f)=\{e \in$ $\left.\left.E A \mid \varphi(e)=f, \partial_{0}(e)=a\right\}\right)$.

Covering morphisms of graphs of groups were originally defined by Bass (Definitions 2.1 and 2.6 of $[\mathrm{B}]$ ). By Proposition 2.7 of $[\mathrm{B}]$, a covering morphism $\Phi: \mathbb{A} \rightarrow \mathbb{A}^{\prime}$ induces a monomorphism of fundamental groups $\Phi_{a_{0}}: \pi_{1}\left(\mathbb{A}, a_{0}\right) \rightarrow \pi_{1}\left(\mathbb{A}^{\prime}, a_{0}^{\prime}\right)$ and an isomorphism of universal covers $\left.\widetilde{\Phi}: \widetilde{\left(\mathbb{A}, a_{0}\right)} \rightarrow \widetilde{\left(\mathbb{A}^{\prime}, a_{0}^{\prime}\right.}\right)$ (where $a_{0} \in V A$ and $a_{0}^{\prime}=\varphi\left(a_{0}\right)$ for $\varphi: A \rightarrow A^{\prime}$ the underlying graph morphism of the covering $\Phi)$.

We will embed cocompact lattices in a Kac-Moody group $G$ by constructing coverings of the corresponding graphs of groups. Our first example shows that free groups may be embedded as cocompact lattices in $G$. 
Proposition 9. Let $G$ be a locally compact rank 2 affine or hyperbolic Kac-Moody group over $\mathbb{F}_{q}$. Let $X=X_{q+1}$ be the Tits building of $G, a(q+1)$-regular tree. Let $A$ be any $(q+1)$-regular bipartite graph. Then

(1) The free group $F_{s}=\pi_{1}(A)$ is a discrete subgroup of $G$, where $s$ is the number of edges outside any maximal tree in $A$.

(2) If $A$ is finite, then the free group $F_{s}=\pi_{1}(A)$ is a cocompact lattice in $G$, where $s$ is the number of edges outside any maximal tree in $A$.

To prove this, we construct a covering morphism of graphs of groups. We need the following lemma regarding embedding lattices in $G$.

Lemma 10. Let $G$ be a locally compact rank 2 affine or hyperbolic Kac-Moody group over $\mathbb{F}_{q}$. Let $\mathbb{A}=\left(A, \mathcal{A}_{v}, \mathcal{A}_{e}, \alpha_{e}\right)$ be any graph of groups. Let $\mathbb{G}$ be the graph of groups for $G$. Suppose there is a covering morphism $\Phi: \mathbb{A} \rightarrow \mathbb{G}$. Then

(1) $\Gamma=\pi_{1}(\mathbb{A})$ is a subgroup of $G=\pi_{1}(\mathbb{G})$.

(2) If $\mathbb{A}$ is a graph of finite groups, then $\Gamma=\pi_{1}(\mathbb{A})$ is a discrete subgroup of $G$.

(3) If $\mathbb{A}$ is a finite graph of finite groups, then $\Gamma=\pi_{1}(\mathbb{A})$ is a cocompact lattice in $G$.

(4) If $\mathbb{A}$ is an infinite graph of finite groups, with $\operatorname{Vol}(\mathbb{A}):=\sum_{v \in V A} \frac{1}{\left|\mathcal{A}_{v}\right|}<\infty$, then $\Gamma=$ $\pi_{1}(\mathbb{A})$ is a nonuniform lattice in $G$.

Proof. (1) is a restatement of Bass's equivalence between a covering morphism of graphs of groups and an embedding of fundamental groups of graphs of groups ([B], Proposition 2.7).

For (2), let $G_{0}$ be the faithful quotient of $G$ on $X$. By (1), $\Gamma$ is a subgroup of $G$, and hence acts on $X$. Since $\mathbb{A}$ is a graph of finite groups, the action has finite vertex stabilizers, and thus $\Gamma$ is discrete in $\operatorname{Aut}(X)$. Moreover, $G_{0}$ is a closed subgroup of $A u t(X)$ and the quotient topology on $G_{0}$ coincides with the quotient topology on $A u t(X)$ ( $[\mathrm{CG}]$, Sec9). Therefore $\Gamma$ is discrete in Aut $(X)$ if and only if $\Gamma$ is discrete in $G$.

For (3), since $A$ is a finite graph, the map $A=\pi_{1}(\mathbb{A}) \backslash X \longrightarrow G \backslash X$ has finite fibers. Since $\pi_{1}(\mathbb{A})$ is discrete in $G$ it follows from $([\mathrm{BL}], 1.5)$ that $\pi_{1}(\mathbb{A})$ is cocompact in $G$.

For $(4)$, by $([\mathrm{BL}], 1.6) \operatorname{Vol}(\mathbb{A})<\infty$ if and only if $\mu(\Gamma \backslash G)<\infty$ and

$$
\mu(G \backslash \backslash X):=\frac{1}{\mu\left(P_{1}\right)}+\frac{1}{\mu\left(P_{2}\right)}<\infty,
$$

where $\mu$ is the Haar measure on $G$. Since $\Gamma$ is discrete in $G$ and $\mu(\Gamma \backslash G)<\infty$ and $A$ is an infinite graph, it follows that $\Gamma$ is nonuniform in $G$.

We may now prove Proposition 9 by constructing a covering morphism of graphs of groups. Recall the graph of groups for $G$ :

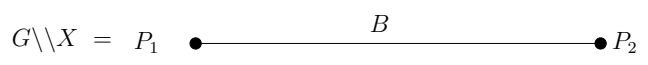


Proof. Let $A$ be any $(q+1)$-regular bipartite graph and let $\mathbb{A}=(A,\{1\},\{1\})$ be the corresponding graph of groups with trivial vertex and edge groups. Then $F_{s}=\pi_{1}(\mathbb{A})$, where $s$ is the number of edges outside any maximal tree of $A$. By the lemma above, it is enough to show that there is a covering morphism $\mathbb{A} \longrightarrow \mathbb{G}$. Since $A$ is bipartite there is a graph morphism from $A$ to the simplex (the underlying graph of $\mathbb{G}$ ). For each $a \in V A, e \in E A$, the vertex map $\varphi_{a}$ and the edge map $\varphi_{e}$ are inclusions of the trivial group. Choose $\left\{\delta_{e}\right\}$ to be a complete set of coset representatives of $P_{i} / B$ for $i=1,2$. Since each edge and vertex group is trivial the diagram

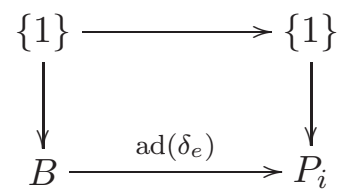

commutes for any $e \in E A$. Moreover, if the quotient graph for $G$ is the single edge $f$ with vertices $a_{1}$ and $a_{2}$, then for $i=1,2$ the map

$$
\coprod_{e \in \varphi_{\left(a_{i}\right)}^{-1}(f)}\{1\}_{e} \longrightarrow P_{i} / B \text { by }\{1\}_{e} \mapsto \delta_{e} B
$$

is map from $q+1$ copies of the trivial group (because $A$ is $(q+1)$-regular) to distinct cosets of $P_{i} / B$. Thus the map is bijective, and the desired covering morphism is obtained.

3.3. Extending Coverings of Edge-indexed Graphs to Covering Morphisms of Graphs of Groups. In the last section we described a technique for embedding fundamental groups of graphs of groups by constructing covering morphisms of graphs of groups. This technique requires us to find an infinite family of elements $\delta_{e}$ that produce corresponding commutative diagrams and bijections on cosets.

In some cases it is possible to construct a simpler covering of edge-indexed graphs (see [BL]),

$$
p:(B, j) \longrightarrow(A, i) \text {. }
$$

Here $p: B \longrightarrow A$ is a graph morphism such that for all $e \in E A, \partial_{0}(e)=a$, and $b \in p^{-1}(a)$, we have

$$
i(e)=\sum_{f \in p_{(b)}^{-1}(e)} j(f),
$$

where $p_{(b)}: E_{0}^{B}(b) \longrightarrow E_{0}^{A}(a)$ is the local map on stars $E_{0}^{B}(b)$ and $E_{0}^{A}(a)$ of vertices $b \in V B$ and $a \in V A$. If $b \in V B, p(b)=a \in V A$, then we can identify

$$
(\widetilde{A, i, a})=X=(\widetilde{B, j, b})
$$

so that the diagram of natural projections

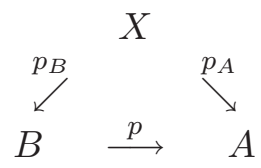

commutes.

Let $\varphi:(A, i) \rightarrow\left(A^{\prime}, i^{\prime}\right)$ be a covering of edge-indexed graphs. In this section we consider the following natural question: 
Question 11. Are there faithful finite groupings $\mathbb{A}$ and $\mathbb{A}^{\prime}$ of $(A, i)$ and $\left(A^{\prime}, i^{\prime}\right)$ respectively such that $\varphi$ extends to a covering morphism $\Phi: \mathbb{A} \longrightarrow \mathbb{A}^{\prime}$ ?

A positive answer to Question 11 would give rise to a pair $\Gamma \leq \Gamma^{\prime}$ of discrete subgroups of $\operatorname{Aut}(X)$, where $X=\widetilde{(A, i)}=\widetilde{\left(A^{\prime}, i^{\prime}\right)}$ is the universal covering tree, and $\Gamma=\pi_{1}(\mathbb{A}, a)$ and $\Gamma^{\prime}=\pi_{1}\left(\mathbb{A}^{\prime}, a^{\prime}\right)$ are the respective fundamental groups, with basepoints $a \in V A$ and $a^{\prime}=\varphi(a)$.

In Theorem 12 we give a sufficient condition for Question 11 to have a positive answer in the case that $\mathbb{A}$ and $\mathbb{A}^{\prime}$ are abelian groupings. In this case, the definition of a covering morphism is simplified as follows.

Let $\Phi: \mathbb{A} \rightarrow \mathbb{A}^{\prime}$ be a covering morphism, as in Definition 1 , with monomorphisms

$$
\varphi_{a}: \mathcal{A}_{a} \rightarrow \mathcal{A}_{\varphi(a)}^{\prime} \quad(a \in A), \quad \varphi_{e}=\varphi_{\bar{e}}: \mathcal{A}_{e} \rightarrow \mathcal{A}_{\varphi(e)}^{\prime} \quad(e \in E A),
$$

and conjugating elements $\delta_{e} \in \mathcal{A}_{\varphi(a)}^{\prime}\left(\right.$ where $\left.\partial_{0} e=a\right)$.

Consider the case where each action $\operatorname{ad}\left(\delta_{e}\right)$ is trivial. This must occur in particular when the groups $\mathcal{A}_{\varphi(a)}^{\prime}$ are abelian. Since the maps $\varphi_{a}$ and $\varphi_{e}$ are monomorphisms, we may identify the groups $\mathcal{A}_{a}$ and $\mathcal{A}_{e}$ with their images in $\mathcal{A}_{\varphi(a)}^{\prime}$ and $\mathcal{A}_{\varphi(e)}^{\prime}$ respectively. Condition (3a) of Definition 1 then becomes

$$
\left(3 \mathrm{a}^{\prime}\right) \quad \alpha_{e}=\left.\alpha_{\varphi(e)}^{\prime}\right|_{\mathcal{A}_{e}} .
$$

We have the following.

Theorem 12. Let $\varphi:(A, i) \rightarrow\left(A^{\prime}, i^{\prime}\right)$ be a covering of edge-indexed graphs. Let $\mathbb{A}$ and $\mathbb{A}^{\prime}$ be finite abelian groupings of $(A, i)$ and $\left(A^{\prime}, i^{\prime}\right)$ respectively. Suppose further that

(1) For all $a \in V A$ and $e \in E A$, we have $\mathcal{A}_{a} \leq \mathcal{A}_{\varphi(a)}^{\prime}$ and $\mathcal{A}_{e} \leq \mathcal{A}_{\varphi(e)}^{\prime}$.

(2) For all $e \in E A$, we have $\alpha_{e}=\alpha_{\varphi(e)}^{\prime} \mid \mathcal{A}_{e}$.

(3) For all $a \in V A$ and $f \in E A^{\prime}$ such that $\partial_{0} f=\varphi(a)$, and for all $e \in \varphi_{(a)}^{-1}(f)$, we have $\mathcal{A}_{a} \cap \alpha_{f}^{\prime} \mathcal{A}_{f}^{\prime}=\alpha_{e} \mathcal{A}_{e}$.

Then $\varphi$ extends to a covering morphism $\Phi=(\varphi,(\delta)): \mathbb{A} \longrightarrow \mathbb{A}^{\prime}$.

Proof We define the monomorphisms $\varphi_{a}$ and $\varphi_{e}$ to be just the inclusion maps. We have seen that since we are using abelian groups, condition (3a) in the definition of a covering morphism reduces to condition (2) in the statement of this theorem. It remains only to define the elements $\delta_{e}$ for each $e \in E A$ and show that condition (3b) in the definition of a covering morphism is satisfied. So let $a \in V A$ and let $f \in E A^{\prime}$ be such that $\partial_{0} f=\varphi(a)$. We claim that the $\left\{\delta_{e}\right\}_{e \in \varphi_{(a)}^{-1}(f)}$ may be chosen as distinct coset representatives of

$$
\left(\mathcal{A}_{\varphi(a)}^{\prime} / \alpha_{f}^{\prime} \mathcal{A}_{f}^{\prime}\right) /\left(\mathcal{A}_{a} \alpha_{f}^{\prime} \mathcal{A}_{f}^{\prime} / \alpha_{f}^{\prime} \mathcal{A}_{f}^{\prime}\right)
$$

Note that since $\mathcal{A}_{\varphi(a)}^{\prime}$ is an abelian group, its subgroups $\mathcal{A}_{a}$ and $\alpha_{f}^{\prime} \mathcal{A}_{f}^{\prime}$ are normal, and so $\mathcal{A}_{a} \alpha_{f}^{\prime} \mathcal{A}_{f}^{\prime}$ is a subgroup.

We first show that the group $\mathcal{A}_{a} / \alpha_{e} \mathcal{A}_{e}$ naturally injects to a subgroup of the group $\mathcal{A}_{\varphi(a)}^{\prime} / \alpha_{f}^{\prime} \mathcal{A}_{f}^{\prime}$. Consider the map

$$
\mathcal{A}_{a} / \alpha_{e} \mathcal{A}_{e} \rightarrow \mathcal{A}_{\varphi(a)}^{\prime} / \alpha_{f}^{\prime} \mathcal{A}_{f}^{\prime}
$$

which takes $[s]_{e}$ to $[s]_{f}$, where $s \in \mathcal{A}_{a}$. This map is well-defined since by (1) and (2) above, $\alpha_{e} \mathcal{A}_{e}$ is a subgroup of $\alpha_{f}^{\prime} \mathcal{A}_{f}^{\prime}$. It is clearly a homomorphism, and by condition (3) in the statement of 
this theorem it is in fact a monomorphism. The image of $\mathcal{A}_{a} / \alpha_{e} \mathcal{A}_{e}$ under this monomorphism is the group $\mathcal{A}_{a} \alpha_{f}^{\prime} \mathcal{A}_{f}^{\prime} / \alpha_{f}^{\prime} \mathcal{A}_{f}^{\prime}$.

We must also show that the number of cosets in the quotient group in (1) above is precisely the number of edges $e \in \varphi_{(a)}^{-1}(f)$. Recall that if $K$ and $L$ are subgroups of an abelian group $H$ then the index $[H / K: L K / K]$ equals $[H: K] /[L: K \cap L]$. Applying this with $H=\mathcal{A}_{\varphi(a)}^{\prime}, K=\alpha_{f}^{\prime} \mathcal{A}_{f}^{\prime}$ and $L=\mathcal{A}_{a}$, and using condition (3) in the statement of this theorem, we find that for any $e \in \varphi_{(a)}^{-1}(f)$ the number of cosets in the quotient group $(1)$ is $i^{\prime}(f) / i(e)$. Thus the indexing $i$ is constant on edges $e \in \varphi_{(a)}^{-1}(f)$. Since $\varphi$ is a covering of edge-indexed graphs, we have

$$
i^{\prime}(f)=\sum_{e \in \varphi_{(a)}^{-1}(f)} i(e) .
$$

Hence the number of edges in $\varphi_{(a)}^{-1}(f)$ must be exactly $i^{\prime}(f) / i(e)$ for any $e \in \varphi_{(a)}^{-1}(f)$ as well.

The map $\Phi_{a / f}$ is then just the map that for each $e \in \varphi_{(a)}^{-1}(f)$ sends the group $\mathcal{A}_{a} / \alpha_{e} \mathcal{A}_{e}$ to the $\operatorname{coset}\left(\mathcal{A}_{a} \alpha_{f}^{\prime} \mathcal{A}_{f}^{\prime} / \alpha_{f}^{\prime} \mathcal{A}_{f}^{\prime}\right) \delta_{e}$. This is bijective since we have exactly one representative from each coset.

\section{Existence of COCOMPaCt Lattices in RANK 2 KaC-Moody Groups}

Recall that a rank 2 Kac-Moody group $G$ over a finite field $\mathbb{F}_{q}$ acts on the tree $X=X_{q+1}$ with quotient a simplex. The quotient graph of groups is given in Figure 4.

$$
G \backslash \backslash X=P_{1} \bullet \quad B \quad \bullet P_{2}
$$

FiguRE 4. graph of groups for a rank 2 Kac-Moody group (also Figure 3)

As discussed in section 2.4, $B$ is the minimal parabolic subgroup, and the Weyl group is given by

$$
W=<w_{1}, w_{2}>\cong \mathbb{Z} / 2 \mathbb{Z} * \mathbb{Z} / 2 \mathbb{Z} .
$$

The vertex groups are the standard parabolic subgroups

$$
P_{1}=B \sqcup B w_{1} B, P_{2}=B \sqcup B w_{2} B .
$$

The Kac-Moody group $G$ has the corresponding amalgamated product decomposition $G=$ $P_{1} *_{B} P_{2}$. We restate the following lemma of Lubotzky to give a sufficient condition on the action of an amalgamated product of the form $\Gamma=\mathcal{A}_{1} * \mathcal{A}_{1} \cap \mathcal{A}_{2} \mathcal{A}_{2}$ yielding $\Gamma$ as a cocompact subgroup of $G$. We then use this lemma to construct a cocompact lattice in a rank 2 Kac-Moody group over a field of characteristic 2 .

Lemma 13 ([L], Lemma 3.1). Let $G$ be a rank 2 affine or hyperbolic Kac-Moody group over the field $\mathbb{F}_{q}$ and let $X=X_{q+1}$ be the Bruhat-Tits tree of $G$. Let $x_{1}, x_{2}$ be adjacent vertices of $X$ and let $\mathcal{A}_{1}$ and $\mathcal{A}_{2}$ be finite subgroups of $G$ such that

(1) $\mathcal{A}_{i}$ fixes $x_{i}$ for $i=1,2$, and $\operatorname{Stab}_{\mathcal{A}_{i}}\left(x_{3-i}\right)=\mathcal{A}_{1} \cap \mathcal{A}_{2}$.

(2) For $i=1,2 \mathcal{A}_{i}$ acts transitively on the $q+1$ neighbors of $x_{i}$.

Then the group $\Gamma=\mathcal{A}_{1} * \mathcal{A}_{1} \cap \mathcal{A}_{2} \mathcal{A}_{2}$ is a cocompact lattice in $G$. 
Recall that the subgroups $M_{q}$ in Lemma 2 ([L], Lemma 3.5) act transitively on the projective line. Moreover the group $P_{i}$ has Levi factor $L_{i}=\left(S L_{2}\left(\mathbb{F}_{q}\right) \times\left(\mathbb{F}_{q}^{\times}\right)\right)_{i}$ where $L_{i}$ is the group generated by $H \cong \mathbb{F}_{q}^{\times} \times \mathbb{F}_{q}^{\times}$and the root groups $U_{ \pm \alpha_{i}}$. By a slight abuse of notation, we let $M_{q}$ denote the image of the group $M_{q}$ of Lemma 2 in the Levi factor $L_{1}$ and we let $\widetilde{M}_{q}$ denote the image of $M_{q}$ in $L_{2}$. It is then clear that $M_{q}$ fixes $x_{1}$ and $\widetilde{M}_{q}$ fixes $x_{2}$. Since $M_{q}$ and $\widetilde{M}_{q}$ each act transitively on $\mathbb{P}_{q+1}$, it follows from the construction of $X$ that $M_{q}$ (resp. $\widetilde{M}_{q}$ ) acts transitively on the $q+1$ neighbors of $x_{1}$ (resp. neighbors of $x_{2}$ ). Then Lemma 14 follows directly from Lemma 13.

Lemma 14. Let $G$ be a locally compact rank 2 affine or hyperbolic Kac-Moody group over $\mathbb{F}_{q}$. Let $x_{1}$ and $x_{2}$ be adjacent vertices in the Tits building $X=X_{q+1}$ such that $P_{i}=\operatorname{Stab}\left(x_{i}\right)$ for $i=1,2$. Let $M_{q}$ and $\widetilde{M}_{q}$ be subgroups of $P_{1}$ and $P_{2}$ as described above. If $\operatorname{Stab}_{M_{q}}\left(x_{2}\right)=$ $M_{q} \cap \widetilde{M}_{q}=\operatorname{Stab}_{\widetilde{M}_{q}}\left(x_{1}\right)$, then

is a cocompact lattice in $G$.

$$
M_{q} *_{M_{q} \cap \widetilde{M_{q}}} \widetilde{M_{q}}
$$

Corollary 15. Let $G$ be a locally compact rank 2 affine or hyperbolic Kac-Moody group over $\mathbb{F}_{q}$, and suppose $q=2^{s}$. Let $M_{q}$ and $\widetilde{M}_{q}$ be as in Lemma 14. Then

(1) $\operatorname{Stab}_{M_{q}}\left(x_{2}\right)=\operatorname{Stab}_{\widetilde{M}_{q}}\left(x_{1}\right)=\{1\}$

(2) $M_{q} * \widetilde{M}_{q}$ is a cocompact lattice in $G$.

Proof. Note that the star in $X$ of $x_{1}$, denoted $\operatorname{Star}_{X}\left(x_{1}\right)$ consists of the $q+1$ edges with initial vertex $x_{1}$. Moreover $M_{q}$ has order $q+1$ and acts transtively on $\operatorname{Star}_{X}\left(x_{1}\right)=\mathbb{P}^{1}\left(\mathbb{F}_{q}\right)$ by Lemma 2 . By transitivity of $M_{q}$ on $\operatorname{Star}_{X}\left(x_{1}\right)$, the orbit of the edge $\left(x_{1}, x_{2}\right)$ has cardinality $q+1$. By the orbit-stabilizer theorem it follows that the stabilizer of $\left(x_{1}, x_{2}\right)$ in $M_{q}$ is trivial. The group $M_{q}$ fixes $x_{1}$, and hence $\operatorname{Stab}_{M_{q}}\left(x_{2}\right)=\{1\}$. A similar argument shows that $\operatorname{Stab}_{\bar{M}_{q}}\left(x_{1}\right)=\{1\}$. Thus $\operatorname{Stab}_{M_{q}}\left(x_{2}\right)=\operatorname{Stab}_{\widetilde{M}_{q}}\left(x_{1}\right)=M_{q} \cap \widetilde{M}_{q}=\{1\}$, and we have proven (1). Part (2) now follows immediately from Lemma 14.

A similar line of argument was used in Section 6.1 of $[\mathrm{LW}]$ to embed $\mathbb{Z} /(q+1) \mathbb{Z} * \mathbb{Z} /(q+1) \mathbb{Z}$ as a cocompact lattice in $S L_{2}\left(\mathbb{F}_{q}\left(\left(t^{-1}\right)\right)\right)$ for $q=2^{s}$.

When $q=2$, we have $M_{q} \cong \widetilde{M}_{q} \cong \mathbb{Z} / 3 \mathbb{Z}$. The following corollary gives the cocompact lattice subgroup $\Gamma$ with quotient a simplex introduced in Theorem 1.

Corollary 16. Let $G$ be a locally compact rank 2 affine or hyperbolic Kac-Moody group over $\mathbb{F}_{2}$. Then $\mathbb{Z} / 3 \mathbb{Z} * \mathbb{Z} / 3 \mathbb{Z}$ is a cocompact lattice in $G$.

Remark: Note that the two factors of the cocompact lattice in the corollary are distinct copies of $\mathbb{Z} / 3 \mathbb{Z}$ with trivial intersection. In fact it is possible to show that

$$
M_{2} \cong<\chi_{1}(1) \chi_{-1}(1)>\text { and } \widetilde{M}_{2} \cong<\chi_{2}(1) \chi_{-2}(1)>,
$$

where $\chi_{ \pm i}(1) \in P_{i}-B$. For simplicity we just write $\mathbb{Z} / 3 \mathbb{Z}$ for $M_{2}$ and $\widetilde{M}_{2}$.

For the remainder of this section, let $G$ be a rank 2 affine or hyperbolic Kac-Moody group over a finite field $F_{q}$, and consider a cocompact lattice $\Gamma$ of the form $M_{q} *_{M} \widetilde{M}_{q}$. The main idea 
is to now use covering theory to construct further cocompact lattices of $G$ as subgroups of $\Gamma$. More precisely, we seek to exhibit edge-indexed graphs $(B, j)$ for which a covering $p:(B, j) \rightarrow$ $(A, i)$ exists, where $(A, i)$ is the edge-indexed graph corresponding to a previously constructed cocompact lattice, such as $\mathbb{Z} / 3 \mathbb{Z} * \mathbb{Z} / 3 \mathbb{Z}$. We then extend the coverings to covering morphisms $\varphi: \mathbb{B} \rightarrow \mathbb{A}$ of graphs of groups, yielding an embedding of fundamental groups $\pi_{1}(\mathbb{B}) \rightarrow \pi_{1}(\mathbb{A})$.

The following lemma carries out this strategy of extending coverings of edge-indexed graphs more generally. In particular we construct subgroups of an arbitrary amalgamated free product $\Gamma=\mathcal{A}_{1} * \mathcal{A}_{3} \mathcal{A}_{2}$, where $\mathcal{A}_{3}$ embeds in $\mathcal{A}_{1}$ and $\mathcal{A}_{2}$ as a finite-index subgroup. The construction uses Theorem 12 in Section 3.3 to extend coverings of edge-indexed graphs. This necessitates the condition that the groups $\mathcal{A}_{1}$ and $\mathcal{A}_{2}$ are abelian. From this lemma we then specialize to the Kac-Moody setting.

Lemma 17. Let $\Gamma=\mathcal{A}_{1} * \mathcal{A}_{3} \mathcal{A}_{2}$ be a group, and suppose that $\left[\mathcal{A}_{1}: \mathcal{A}_{3}\right]=a<\infty$ and $\left[\mathcal{A}_{2}\right.$ : $\left.\mathcal{A}_{3}\right]=b<\infty$. Let $X$ be the locally finite tree on which $\Gamma$ acts with quotient a simplex. Suppose further that $\mathcal{A}_{1}$ and $\mathcal{A}_{2}$ are abelian. Then $\Gamma_{1}=\underset{\substack{\mathcal{\mathcal { A } _ { 3 }} \\ k=1, \ldots, a}}{*}\left(\mathcal{A}_{2}\right)_{k}$ is a subgroup of $\Gamma$. Moreover, if $\mathcal{A}_{2}$ is finite then $\Gamma_{1}$ is a cocompact lattice in $\Gamma$.

Proof. Let $X$ be the $(a, b)$ bihomogeneous tree on which $\Gamma=\mathcal{A}_{1} * \mathcal{A}_{3} \mathcal{A}_{2}$ acts with quotient a simplex. Let $(A, i)=(\Gamma \backslash X, i)$ be the resulting edge-indexed graph, and let $(B, j)$ be the edge-indexed 'a-star' shown in Figure 5.

The graph morphism $p:(B, j) \rightarrow(A, i)$ depicted is a covering of edge-indexed graphs. Assume without loss of generality that $\mathcal{A}_{3} \leq \mathcal{A}_{1}$, and let $\alpha: \mathcal{A}_{3} \rightarrow \mathcal{A}_{2}$ be a monomorphism. Let $\mathbb{A}$ be the graph of groups associated with the action of $\Gamma$ on $X$. Give $(B, j)$ the abelian grouping $\mathbb{B}$ as shown in Figure 6.

It is straightforward to check that the abelian groupings $\mathbb{A}$ and $\mathbb{B}$, together with the edgeindexed covering $p$, satisfy the sufficient conditions listed in Theorem 12. Therefore $p$ extends to a covering morphism $\varphi: \mathbb{B} \rightarrow \mathbb{A}$ of graphs of groups.

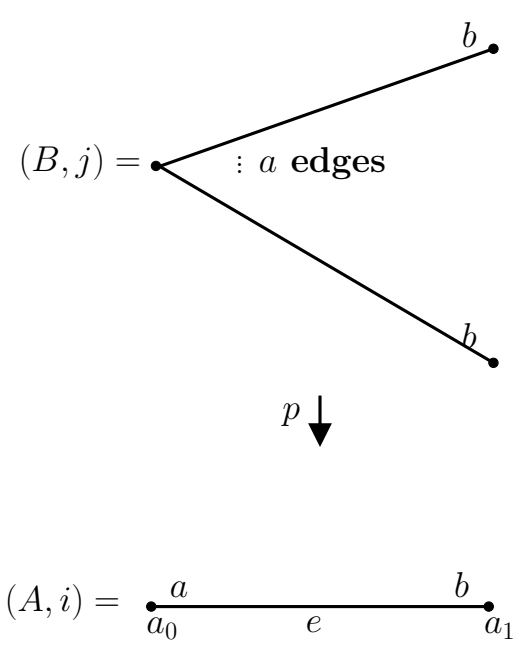

Figure 5. edge-indexed covering of the simplex by the $a$-star 

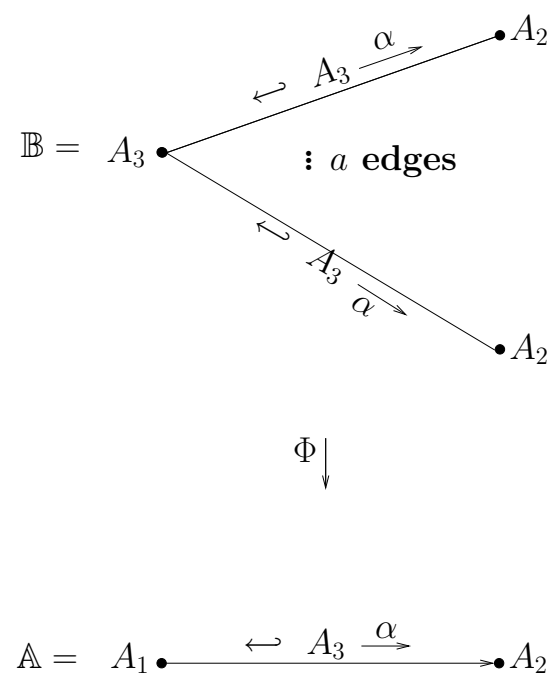

FIGURE 6. abelian grouping of the $a$-star, covering the simplex grouping

By Proposition 2.7 of $[\mathrm{B}]$ it follows that $\pi_{1}(\mathbb{B})=\underset{\substack{\mathcal{A}_{3} \\ k=1, \ldots, a}}{*}\left(\mathcal{A}_{2}\right)_{k}$ embeds in $\pi_{q}(\mathbb{A})=\Gamma$. If $\mathcal{A}_{2}$ is finite then $\underset{\substack{\mathcal{A}_{3} \\ k=1, \ldots, a}}{*}\left(\mathcal{A}_{2}\right)_{k}$ is a cocompact lattice in $\Gamma$.

Given that the rank 2 Kac-Moody group $G$ has a cocompact lattice whose quotient is a simplex, we now have a sufficient condition yielding a ' $q+1$-star' which embeds in $G$ as a cocompact lattice.

Corollary 18. Let $G$ be a locally compact rank 2 affine or hyperbolic Kac-Moody group over $\mathbb{F}_{q}$, and suppose that $\mathcal{A}_{1} * \mathcal{A}_{3} \mathcal{A}_{2}$ is a cocompact lattice in $G$ with quotient a simplex. Suppose further that $\mathcal{A}_{1}$ and $\mathcal{A}_{2}$ are abelian. Then

is a cocompact lattice in $G$.

$$
\underset{k=1, \ldots, q+1}{\stackrel{*}{\mathcal{A}}}\left(\mathcal{A}_{2}\right)_{k}
$$

In particular, we have an embedding of such a $q+1$-star when $\mathbb{F}_{q}$ is a field of characteristic 2 if the subgroups $M_{q}$ and $\widetilde{M}_{q}$ are abelian.

Corollary 19. Let $G$ be a locally compact rank 2 affine or hyperbolic Kac-Moody group over $\mathbb{F}_{q}$, and suppose $q=2^{s}$. Let $M_{q} * \widetilde{M}_{q}$ be the cocompact lattice given in Corollary 15. If $M_{q}$ and $\widetilde{M}_{q}$ are abelian, then

is a cocompact lattice in $G$.

$$
k_{k=1, \ldots, q+1}^{*}\left(\widetilde{M_{q}}\right)_{k}
$$


In particular the Corollary holds when $q=2$. This yields the cocompact lattice which is the second subgroup in the infinite descending chain in Theorem 1 (3).

Corollary 20. Let $G$ be a locally compact rank 2 affine or hyperbolic Kac-Moody group over $\mathbb{F}_{2}$. Then

$$
\Gamma_{1} \cong \mathbb{Z} / 3 \mathbb{Z} * \mathbb{Z} / 3 \mathbb{Z} * \mathbb{Z} / 3 \mathbb{Z}
$$

is a cocompact lattice in $G$.

Remark: Note that each copy of $\mathbb{Z} / 3 \mathbb{Z}$ in the graph of groups $\mathbb{B}$ is identical to the image subgroup $\widetilde{M}_{2} \cong<\chi_{2}(1) \chi_{-2}(1)>$ in $\mathbb{A}$ (see remark following the proof of Corollary 16).

There are infinitely many cocompact lattices of $G$ which may be constructed using the technique described in this section. In particular in Section 8 we give the general construction for an infinite descending chain of subgroups of an amalgamated free product and provide an example in the Kac-Moody setting.

\section{Rank 3 Complex of Groups}

Let $G$ be a rank 3 Kac-Moody group of type $\infty$ over the field $\mathbb{F}_{2}$, and let $X$ denote the corresponding Tits building. As described in section 2.4, $X$ is a hyperbolic building whose standard apartment consists of a tiling of the hyperbolic plane by ideal triangles. The full building is constructed by gluings of a third triangle at each edge of the plane. The vertices of the complex are given by cosets of the maximal standard parabolic subgroups:

$$
P_{i, i+1 \bmod 3}=\underset{w \in\left\langle w_{i}, w_{(i+1) \bmod 3}\right\rangle}{\bigsqcup} B w B, i=1, \ldots, 3 .
$$

The edges are given by cosets of

$$
Q_{i}=\bigsqcup_{w \in\left\langle w_{i}\right\rangle} B w B=B \sqcup B w_{i} B, i=1, \ldots, 3 .
$$

The faces are given by cosets of $B$. (Here we are, by abuse of notation, writing $w_{i}$ for the element $\left.\widetilde{w}_{i}.\right)$

There are $q+1=3$ faces adjoining each edge. Note that

$$
P_{i-1, i} \cap P_{i, i+1}=Q_{i} \text { and } \cap_{i=1}^{3} Q_{i}=B .
$$

In general each edge coset is the intersection of the corresponding vertex cosets, and each face is the intersection of the adjoining edges cosets (or vertex cosets).

The quotient by the action of $G$ on $X$ is the triangle of groups in Figure 7 .

We note that the triangle of groups in Figure 7 is a (simple) complex of groups, as defined in III.C of $[\mathrm{BH}]$, and the Kac-Moody group $G$ over the field $\mathbb{F}_{2}$ is the corresponding fundamental group.

Lin and Thomas [LT] show that a covering of complexes of groups induces an embedding of the corresponding fundamental groups. We may thus construct a subgroup of a rank 3 Kac-Moody group by constructing a triangle of groups and constructing a covering from this triangle of 


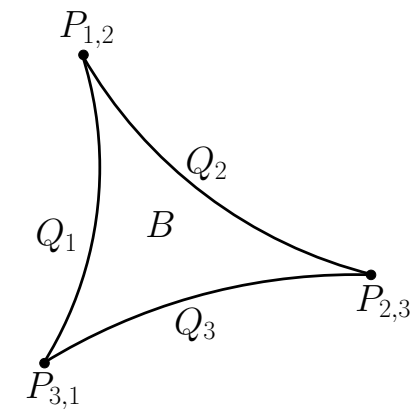

FIGURE 7. quotient triangle of groups for a rank 3 Kac-Moody group

groups to the triangle of groups for $G$. Such a covering of triangles of groups may be constructed by exhibiting the following:

$$
\text { monomorphisms } \varphi_{i j}: \mathcal{A}_{i j} \rightarrow P_{i j}, \quad \varphi_{i}: \mathcal{A}_{i} \rightarrow Q_{i}, \quad i: \mathcal{A} \rightarrow B
$$

such that these monomorphisms induce bijections

$$
\mathcal{A}_{i j} / \mathcal{A} \rightarrow P_{i j} / B, \quad \mathcal{A}_{i} / \mathcal{A} \rightarrow Q_{i} / B, \quad \text { and } \quad \mathcal{A}_{i j} / \mathcal{A}_{i} \rightarrow P_{i j} / Q_{i}
$$

We use this method in the following section to construct subgroups of the rank 3 Kac-Moody group.

\section{Existence of COCOMPACT LATtices in RANK 3 TyPE $\infty$ KAC-Moody Groups Over $\mathbb{F}_{2}$}

Let $G$ be a symmetrizable locally compact rank 3 Kac-Moody group of type $\infty$ over the field $\mathbb{F}_{2}$, and $X$ its Tits building as described in the previous section. We now use coverings of complexes of groups to construct a non-discrete subgroup whose quotient is also an ideal triangle of groups, that is whose quotient is a simplex. This is the non-discrete subgroup $\Lambda^{\prime}$ in rank 3 introduced in Theorem 5. We thank Anne Thomas for explaining to us how to construct the covering morphism of Lemma 21.

Lemma 21. Let $G$ be a locally compact symmetrizable rank 3 Kac-Moody group of type $\infty$ over the field $\mathbb{F}_{2}$. Let $\Lambda^{\prime}$ be the fundamental group of the ideal triangle depicted below. Then $\Lambda^{\prime}$ is a subgroup of $G$. The image of $\Lambda^{\prime}$ in $G$ is non-discrete.

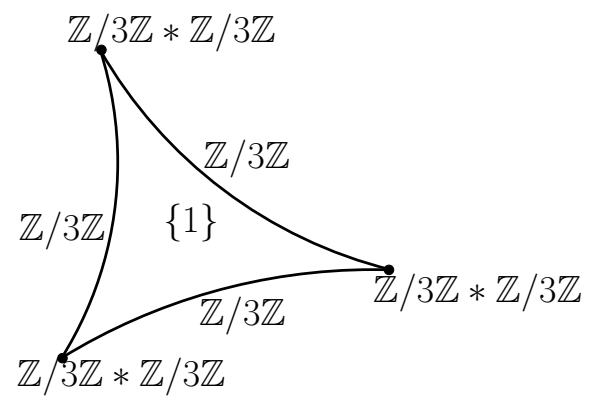

FiguRE 8. triangle of groups for $\Lambda^{\prime}$ 
Proof. We construct a covering of complexes of groups from the triangle of groups for $\Lambda^{\prime}$ to the triangle of groups for $G$.

The face group of $\Lambda^{\prime}$ is trivial so we let the local map $i$ here be the natural inclusion of the identity element into $B$. For the edge groups, write $\mathbb{Z} / 3 \mathbb{Z}=\langle\varepsilon\rangle$ for the cyclic group of order 3 . For each $i=1,2,3$ we claim that there is a group monomorphism $\varphi_{i}:\langle\varepsilon\rangle \rightarrow Q_{i}$.

By definition of the elements $w_{i}=\widetilde{w}_{i}$, and using the fact that in the field $\mathbb{F}_{2}$ we have $1=-1$,

$$
\chi_{i}(1) \chi_{-i}(1)=w_{i} \chi_{i}(1)^{-1} .
$$

Since $\chi_{i}(1)=\chi_{\alpha_{i}}(1) \in U_{\alpha_{i}} \subseteq U \subseteq B$, we have that

$$
\varphi_{i}(\varepsilon)=w_{i} \chi_{i}(1)^{-1} \in B w_{i} B=Q_{i}-B
$$

as required. In particular, $\varphi_{i}(\varepsilon) \neq 1$ and $\left.\left\langle\varphi_{i}(\epsilon)\right\rangle \cap<\varphi_{j}(\epsilon)\right\rangle=\{1\}$ for $i \neq j$.

Next we show that $\varphi_{i}(\varepsilon)$ has order 3 . For this, it is enough to show that $\left(\varphi_{i}(\varepsilon)\right)^{3}=1$. We compute

$$
\begin{aligned}
\left(\varphi_{i}(\varepsilon)\right)^{3} & =\chi_{i}(1) \chi_{-i}(1) \chi_{i}(1) \chi_{-i}(1) \chi_{i}(1) \chi_{-i}(1) \\
& =w_{i} \chi_{-i}(1) \chi_{i}(1) \chi_{-i}(1) \\
& =w_{i} \chi_{-i}(1) w_{i}^{-1} w_{i} \chi_{i}(1) w_{i}^{-1} w_{i} \chi_{-i}(1) w_{i}^{-1} w_{i} \\
& =\chi_{i}(1) \chi_{-i}(1) \chi_{i}(1) w_{i} \\
& =w_{i}^{2} \\
& =1
\end{aligned}
$$

We thus have a monomorphism

$$
\varphi_{i}: \mathbb{Z} / 3 \mathbb{Z} \rightarrow Q_{i}
$$

for each $i=1,2,3$. Moreover, the elements $\left\{\varphi_{i}(1), \varphi_{i}(\varepsilon), \varphi_{i}\left(\varepsilon^{2}\right)\right\}$ form a set of coset representatives of $Q_{i} / B$ since $\varphi_{i}(\varepsilon)$ has order 3 and does not lie in $B$.

For the vertex groups, since $\varphi_{i}(\varepsilon) \in Q_{i}-B$, we have that for $i=1,2,3$,

$$
\varphi_{i}(\langle\varepsilon\rangle) \cap \varphi_{i+1}(\langle\varepsilon\rangle)=\{1\} .
$$

Hence we obtain an embedding

$$
\langle\varepsilon\rangle *\langle\varepsilon\rangle \hookrightarrow P_{i, i+1}
$$

Moreover the image $\varphi_{i}(\langle\varepsilon\rangle)$ in $P_{i, i+1}$ forms a set of coset representatives for $P_{i, i+1} / Q_{i+1}$, and similarly for the image of $\varphi_{i+1}(\langle\varepsilon\rangle)$ and $P_{i, i+1} / Q_{i}$. Finally the image of $\langle\varepsilon\rangle *\langle\varepsilon\rangle$ in $P_{i, i+1}$ forms a set of coset representatives for $P_{i, i+1} / B$ since this image only intersects $B$ trivially.

We have thus constructed a covering of complexes of groups from the complex of groups for $\Lambda^{\prime}$ to the complex of groups for $G$. It follows that $\Lambda^{\prime}$ is a subgroup of $G$. Since the vertex groups are infinite groups, the image of $\Lambda^{\prime}$ in $G$ is non-discrete.

In the following section, we provide a different embedding of $\mathbb{Z} / 3 \mathbb{Z} * \mathbb{Z} / 3 \mathbb{Z} * \mathbb{Z} / 3 \mathbb{Z}$ as a cocompact lattice in $G$. 


\section{ACtions of COCOMPACT LATtiCES ON IDEAL COMPLEXES AND ON THEIR INSCRIBED \\ TREES}

A locally compact Kac-Moody group $G$ of rank 2, and hence any lattice subgroup, comes equipped with an action on a simplicial tree, the Tits building of $G$.

In [C1] the author showed that all symmetrizable locally compact Kac-Moody groups $G$ of rank 3 noncompact hyperbolic type over finite fields $\mathbb{F}_{q}$ have the Haagerup property, and she exhibited an action of $G$ on a simplicial tree $\mathcal{X}$ where certain lattices act discretely, that is, with finite vertex stabilizers. When $G$ has type $\infty$, the tree $\mathcal{X}$ is the bihomogeneous bipartite tree $\mathcal{X}_{3, q+1}$ ([C1]). When $q=2, \mathcal{X}$ is the homogeneous bipartite tree denoted $\mathcal{X}_{3}$. We can think of $\mathcal{X}$ as the tree of barycenters for the rank 3 building $X$.

Thus we have actions of the rank 2 and rank 3 Kac-Moody groups over the field $\mathbb{F}_{2}$ on the trivalent tree. Let $G$ be a rank 3 symmetrizable locally compact Kac-Moody group of type $\infty$ with Tits building $X$. We showed in Lemma 21 that the non-discrete subgroup $\Lambda^{\prime}$ embeds in $G$. The proof of Lemma 21 also shows that the subgroup generated by the face and edge groups of the triangle of groups $\Lambda^{\prime} \backslash \backslash X$ embeds in the subgroup of $G$ generated by the face and edge groups of the triangle of groups $G \backslash \backslash X$. That is, the $\mathcal{X}$-lattice $\Gamma_{1} \cong \mathbb{Z} / 3 \mathbb{Z} * \mathbb{Z} / 3 \mathbb{Z} * \mathbb{Z} / 3 \mathbb{Z}$ embeds as a cocompact lattice in the subgroup, denoted $\mathcal{Q}$, of $G$ generated by all non-maximal standard parabolic subgroups.

It turns out that $\mathcal{Q}=G$. This is easy to see, since we have $\mathcal{Q}=\left\langle Q_{1}, Q_{2}, Q_{3}\right\rangle$ where $Q_{i}=$ $B \sqcup B w_{i} B$. We claim that $\mathcal{Q}=\sqcup_{w \in W} B w B=G$ where $W=\mathbb{Z} / 2 \mathbb{Z} * \mathbb{Z} / 2 \mathbb{Z} * \mathbb{Z} / 2 \mathbb{Z}$ is the infinite Weyl group $W=\left\langle w_{1}\right\rangle *\left\langle w_{2}\right\rangle *\left\langle w_{3}\right\rangle$. Since $W$ is a free product, the multiplication rule for double cosets gives $B w_{i} B \cdot B w B=B w_{i} w B$ for each $i=1,2,3$. Hence $\mathcal{Q}$ generates the Bruhat decomposition for the whole of $G$.

The edge-indexed graph quotient graph of $\Gamma_{1}$ on $\mathcal{X}$ is inscribed (as a tripod formed by the barycenters) in the ideal triangle which is the fundamental chamber for the Weyl group $W$ of $G$.

Our methods show that $\Gamma_{1}$ also acts discretely and cocompactly on $\mathcal{X}$. The following is a summary of these results.

Theorem 22. Let $G$ be a rank 3 symmetrizable locally compact Kac-Moody group of type $\infty$ over the finite field $\mathbb{F}_{2}$. Let $X$ be the Tits building of $G$ and let $\mathcal{X}=\mathcal{X}_{3}$ be the bihomogeneous bipartite simplicial tree inscribed in $X$. Let $\Gamma_{1} \cong \mathbb{Z} / 3 \mathbb{Z} * \mathbb{Z} / 3 \mathbb{Z} * \mathbb{Z} / 3 \mathbb{Z}$ be as in Corollary 20 . Then $\Gamma_{1}$ is a cocompact $\mathcal{X}$-lattice with edge-indexed quotient a tripod. Moreover the image $\Gamma_{1}^{\prime}$ of $\Gamma_{1}$ in $G$ is a cocompact lattice in $G$.

\section{INFINITE DESCENDING CHAINS OF COCOMPACT LATTICES}

In this section, we consider groups of the form $\Gamma_{1}=\underset{\substack{\mathcal{A}_{3} \\ k=1, \ldots, a}}{\stackrel{*}{*}}\left(\mathcal{A}_{2}\right)_{k}$, an amalgamated free product of copies of a group $\mathcal{A}_{2}$ over a subgroup $\mathcal{A}_{3}$ of finite index. The group $\Gamma_{1} \cong \Gamma_{1}^{\prime} \cong$ $\mathbb{Z} / 3 \mathbb{Z} * \mathbb{Z} / 3 \mathbb{Z} * \mathbb{Z} / 3 \mathbb{Z}$, which embeds in a type- $\infty$ Kac-Moody group over $\mathbb{F}_{2}$ as described in Corollary 20 for rank 2 and Theorem 22 for rank 3, is an example of such an amalgamted free product. 

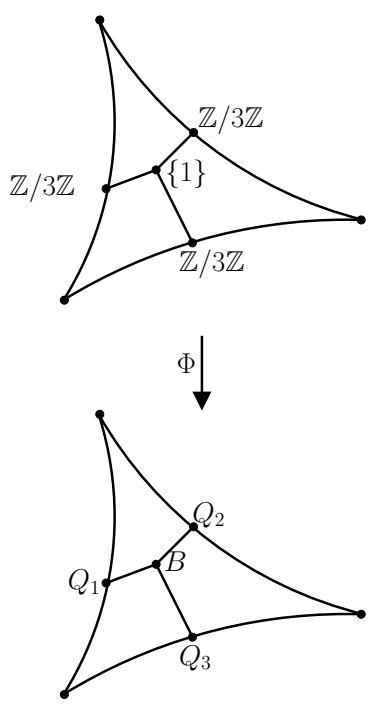

Figure 9 . Embedding of $\mathbb{Z} / 3 \mathbb{Z} * \mathbb{Z} / 3 \mathbb{Z} * \mathbb{Z} / 3 \mathbb{Z}$ in rank 3 group via action on inscribed tree

Remark: The notation reflects the fact that we will be building on the constructions in Lemma 17.

We will construct infinite descending chains of subgroups of $\Gamma_{1}$. Specializing to the Kac-Moody setting, this construction will yield infinite descending chains of subgroups which embed as cocompact lattices in subgroups of a type $\infty$ Kac-Moody group over the field $\mathbb{F}_{2}$. That is we will embed the descending chains in the subgroup $\widetilde{G}$ generated by all standard parabolic subgroups which are disjoint unions of double cosets of $B$ indexed by a single Weyl group generator. In the rank 2 setting $\widetilde{G}$ is the full Kac-Moody group. In the rank 3 setting $\widetilde{G}=\mathcal{Q}$, the subgroup generated by all non-maximal parabolic subgroups (see Theorem 22).

Theorem 23. Let $G$ be a locally compact affine or hyperbolic Kac-Moody group of type $\infty$ over the field $\mathbb{F}_{2}$ with Weyl group $W=<w_{i}>$. Let $\widetilde{G}$ be the subgroup generated by the standard parabolic subgroups of the form $B \sqcup B w_{i} B$. Then $\widetilde{G}$ contains an infinite descending chain $\ldots \Gamma_{3} \leq$

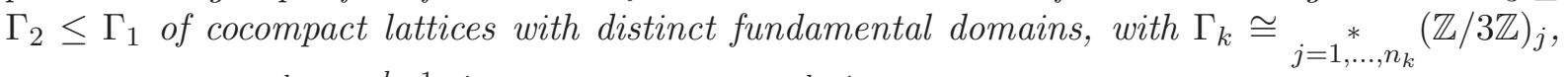
where $n_{k}=1+3^{k}-\sum_{i=0}^{k-1} 3^{i}$ and $\operatorname{Vol}\left(\Gamma_{k}\right)=2(3)^{k-1}$. Hence the $\Gamma_{k}$ are pairwise non-conjugate.

In general we can construct an infinite descending chain of subgroups in $\Gamma_{1}=\underset{\substack{\mathcal{A}_{3} \\ k=1, \ldots, a}}{*}\left(\mathcal{A}_{2}\right)_{k}$ by iterating the technique described in the proof of Lemma 17. We use the action of $\Gamma_{1}$ on an $a$-regular tree $X$. We first build an infinite sequence of coverings of edge-indexed graphs over the quotient of $\Gamma_{1}$ on $X$. We then extend this to an infinite sequence of covering morphisms of finite graphs of finite groups. The following theorem gives the sufficient conditions for these infinite sequences, and Theorem 23 will follow as a special case, with $\mathcal{A}_{2} \cong \mathbb{Z}_{3}$ and $\mathcal{A}_{3} \cong\{1\}$. 
Theorem 24. Let $\mathcal{A}_{2}$ be a group with subgroup $\mathcal{A}_{3}$ of finite index $\left[\mathcal{A}_{2}: \mathcal{A}_{3}\right]=a<\infty$. Let $\Gamma_{1}=\underset{\substack{\mathcal{A}_{3} \\ k=1, \ldots, a}}{*}\left(\mathcal{A}_{2}\right)_{k}$ be an amalgamted free product. Let $X$ be the a-regular tree on which $\Gamma_{1}$ acts with quotient $\Gamma_{1} \backslash X$. Let $(A, i)=\left(\Gamma_{1} \backslash X, i\right)$ denote the edge-indexed quotient graph for $\Gamma_{1}$ on $X$, and let $\mathbb{A}=\Gamma_{1} \backslash \backslash X$ denote the corresponding graph of groups. Suppose that $\mathcal{A}_{2}$ is abelian. Then there exists an infinite sequence of coverings of finite edge-indexed graphs

$$
\cdots \longrightarrow\left(B_{3}, j_{3}\right) \longrightarrow\left(B_{2}, j_{2}\right) \longrightarrow\left(B_{1}, j_{1}\right)=(A, i)
$$

and an infinite sequence of covering morphisms of graphs of groups

$$
\ldots \longrightarrow \mathbb{B}_{3} \longrightarrow \mathbb{B}_{2} \longrightarrow \mathbb{B}_{1}=\mathbb{A}
$$

with $\mathbb{B}_{k}$ a grouping of $\left(B_{k}, j_{k}\right)$ such that $\Gamma_{k+1}=\pi_{1}\left(\mathbb{B}_{k+1}\right) \leq \Gamma_{k}=\pi_{1}\left(\mathbb{B}_{k}\right)$ and $\Gamma_{k} \cong \underset{\substack{\mathcal{\mathcal { A } _ { 3 }} \\ j=0, \ldots, n_{k}}}{*}\left(\mathcal{A}_{2}\right)_{j}$, where $n_{k}=1+a^{k}-\sum_{j=1}^{k-1} a^{j}$. Moreover, if $\left|\mathcal{A}_{2}\right|=c<\infty$, then $\operatorname{Vol}\left(\Gamma_{k}\right)=\frac{2 a^{k}}{c}$. In this case the $\Gamma_{k}$ are pairwise non-conjugate and form a descending chain of cocompact lattices in $\Gamma_{1}$.

To produce the infinite sequence of coverings of finite edge-indexed graphs in Theorem 24, we use an iteration of a method known as 'open fanning' of 'arithmetic bridges' in the edgeindexed graphs in the sequence. This method was used by the first author to prove existence of nonuniform coverings over finite edge-indexed graphs and hence to prove existence of nonuniform lattices on uniform trees ([C2]). This method was also used by Gabriel Rosenberg $([\mathrm{R}])$ to exhibit infinite ascending chains of cocompact lattices with arbitrarily small covolumes in automorphism groups of locally finite trees.

In our edge-indexed graph $(A, i)$, the 'arithmetic bridge' can be taken to be any single separating edge $e$ with an index (ramification factor) $i(e)=\left[\mathcal{A}_{2}: \mathcal{A}_{3}\right]=a$. The open fanning then has the schematic diagram:

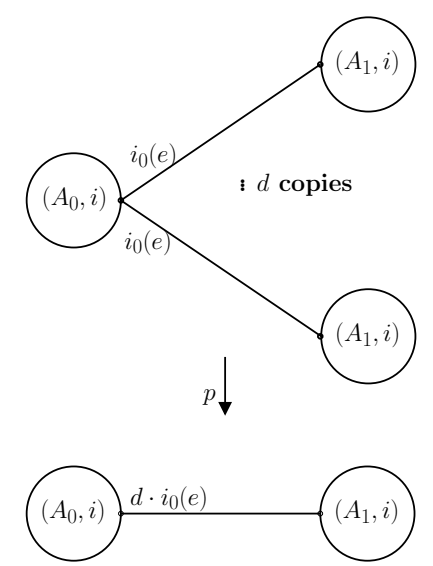

FiguRE 10. schematic of an open fanning along an edge

Proof. (of Theorem 24) We now use the method of open fannings on a single edge to recursively construct a sequence of edge-indexed coverings as follows:

(1) Let $\left(B_{1}, i_{1}\right)=(A, i)=\left(\Gamma_{1} \backslash X, i\right)$ be the edge-indexed ' $a$-star' and choose an edge $e$ of $B_{1}$ with index $i_{1}(e)=i(e)=a$. 
(2) Let $\left(B_{2}, j_{2}\right)$ be an open $a$-fanning on the edge $e$, as shown in Figure 11.

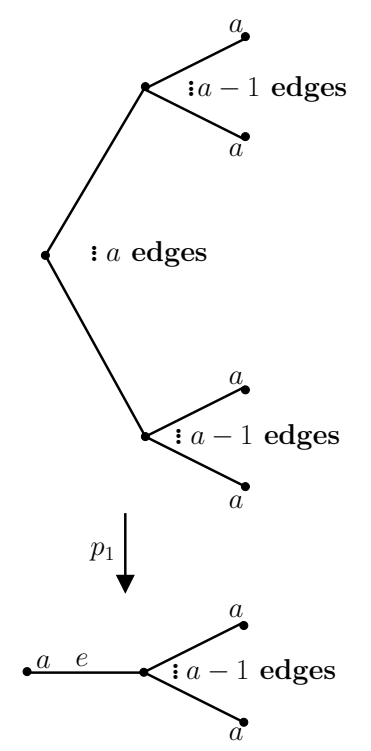

Figure 11. edge-indexed covering $p_{1}:\left(B_{2}, j_{2}\right) \longrightarrow\left(B_{1}, j_{1}\right)$ (open fanning of the $a$-star)

(3) For each $k \geq 2$, choose an edge $e$ of $B_{k}$ with index $i_{k}(e)=a$ and let $\left(B_{k+1}, j_{k+1}\right)$ be an open $a$-fanning of $B_{k}$ on $e$.

Note that the $a$-star $\left(B_{1}, i_{1}\right)$ has $a$ edges of index $a$. An easy induction shows that for $k \geq 1$, $B_{k}$ has

$$
n_{k}=1+a^{k}-\sum_{j=0}^{k-1} a^{j} \geq a \text { edges of index } a .
$$

Thus the recursion is well-defined. Moreover we may associate an abelian grouping $\mathbb{B}_{k}$ to $\left(B_{k}, i_{k}\right)$ consisting of copies of $\mathcal{A}_{2}$ at each initial vertex of these $n_{k}$ edges and copies of $\mathcal{A}_{3}$ at each remaining vertex and along each edge. It is straightforward to check that for $k \geq 1$, the groupings $\mathbb{B}_{k}$ and $\mathbb{B}_{k+1}$, together with the edge-indexed covering $p_{k}:\left(B_{k+1}, i_{k+1}\right) \rightarrow\left(B_{k}, i_{k}\right)$, satisfy the sufficient conditions of Theorem 12 .

Therefore for $k \geq 1, p_{k}$ extends to a covering morphism $\varphi_{k}: \mathbb{B}_{k+1} \rightarrow \mathbb{B}_{k}$ of graphs of groups. By Proposition 2.7 of $[\mathrm{B}]$ it follows that $\Gamma_{k+1}=\pi_{1}\left(\mathbb{B}_{k+1}\right)=\underset{j=1, \ldots, n_{k+1}}{\mathcal{*}} \underset{\mathcal{A}_{3}}{*}\left(\mathcal{A}_{2}\right)_{j}$ embeds in $\Gamma_{k}=\pi_{1}\left(\mathbb{B}_{k}\right)=\underset{\substack{\mathcal{A}_{3} \\ j=1, \ldots, n_{k}}}{*}\left(\mathcal{A}_{2}\right)_{j}$ (and ultimately in $\left.\Gamma_{1}=\pi_{1}\left(\mathbb{B}_{1}\right)\right)$. Thus these $\Gamma_{k}$ form an infinite descending chain of subgroups. If $\left|\mathcal{A}_{2}\right|=c<\infty$, then another easy induction shows for $k \geq 1$,

$$
\operatorname{Vol}\left(\Gamma_{k+1}\right)=a \operatorname{Vol}\left(\Gamma_{k}\right)=2 \frac{a^{k+1}}{c},
$$

where the volume is calculated using Lemma 10 (4). 
As stated previously, Theorem 1 (3) follows from Theorem 24. In particular, the infinite chain of subgroups embed as lattices in the rank 2 Kac-Moody group over $\mathbb{F}_{2}$ and in the rank 3 KacMoody group over $\mathbb{F}_{2}$ of type $\infty$ (see Theorem 22). We also remark that the open fanning of edge-indexed graphs exhibited in the proof above may be viewed in rank 3 within their ambient quotient complex of the Tits building. We illustrate this in Figure 12, showing the fanning from Figure 11 inscribed in the corresponding ideal triangles.

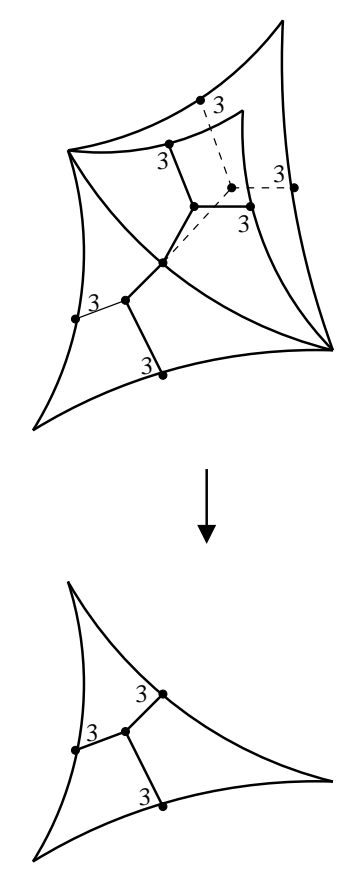

FigURE 12. fanned tripod inscribed in fanned ideal triangles

In constructing the chain of subgroups in Theorem 24, we use an open fanning on a single edge, that is an arithmetic bridge of size 1, at each step. Changing the size of the bridge at any step will yield further (distinct) descending chains. In this manner we can construct an infinite number of infinite descending chains of cocompact lattices. We give additional examples of this construction in [CCR].

\section{REFERENCES}

[B] Bass, H. Covering theory for graphs of groups, J. Pure Appl. Algebra 89 (1993), 3-47.

[BK] Bass, H. and Kulkarni, R. Uniform tree lattices, J. Amer. Math. Soc. 3 (1990), 843-902.

[BL] Bass, H. and Lubotzky, A.Tree lattices. With appendices by H. Bass, L. Carbone, A. Lubotzky, G. Rosenberg and J. Tits, Progress in Mathematics, 176. Birkhaüser Boston, Inc., Boston, MA, (2001). xiv+233 pp.

[Bo1] Bourdon, M. Sur les immeubles fuchsiens et leur type de quasi-isométrie (French) [Fuchsian buildings and their quasi-isometry type] Ergodic Theory Dynam. Systems 20 (2000), no. 2, 343-364.

[Bo2] Bourdon, M. Immeubles hyperboliques, dimension conforme et rigidité de Mostow, Geom. Funct. Anal. 7 (1997), 245-268.

[BP] Bourdon, M. and Pajot, H. Rigidity of quasi-isometries for some hyperbolic buildings Comment. Math. Helv. 75 (2000), no. 4, 701-736. 
[BH] Bridson, M.R. and Haefliger, A. Metric spaces of Non-Positive Curvature, Springer-Verlag, Berlin, 1999.

[CT] Capdeboscq, I. (Korchagina) and Thomas, A. Cocompact lattices of minimal covolume in rank 2 KacMoody groups, Part I: Edge-transitive lattices, arXiv:0907.1350v1 [math.GR]

[C1] Carbone, L. The Haagerup property, Property (T) and the Baum-Connes conjecture for locally compact Kac-Moody groups, To appear in Advances in Algebra (2010).

[C2] Carbone, L. Nonuniform lattices on uniform trees, Memoirs of the American Mathematical Society, vol. 152, no. 724, 127 pages, ISBN 0821827219 (2001).

[CCR] Carbone, L., Cobbs, L. and Rosenberg, G. Tree lattices subgroups, To appear in Groups, Complexity and Cryptology (2010)

[CER] Carbone, L., Ershov, M. and Ritter, G. Abstract simplicity of complete Kac-Moody groups over finite fields, Journal of Pure and Applied Algebra, 212, (2008), 2147-2162.

[CG] Carbone, L. and Garland, H. Existence of Lattices in Kac-Moody Groups over Finite Fields, Communications in Contemporary Math, Vol 5, No.5, (2003), 813-867.

[Co] Cobbs, L. Lattices in Kac-Moody groups, PhD Thesis, Rutgers University (2009).

[DJ] Dymara, J. and Januszkiewicz, T. Cohomology of buildings and of their automorphism groups, Inventiones Math. 150, 3, (2002), 579-627.

[Gr] Gruenberg, K. W. Residual properties of infinite soluble groups, Proc. London Math. Soc. 7 (1957), $29-62$.

[GP] Gaboriau, D. and Paulin, F. Sur les immeubles hyperboliques. (French) [On hyperbolic buildings] Geom. Dedicata 88 (2001), no. 1-3, 153-197.

[GN] Gritsenko, V. A. and Nikulin, V. V Siegel automorphic form corrections of some Lorentzian Kac-Moody Lie algebras, Amer. J. Math. 119 (1997), no. 1, 181-224.

[H] Haefliger, A. Complexes of Groups and Orbihedra, Group Theory from a Geometrical Viewpoint (E. Ghys, A. Haefliger, A. Verjovsky, ed), Proc. ICTP Trieste 1990, World Scientific, Singapore, 1991, 504-540.

[HM] Harvey, J. A. and Moore, G. Algebras, BPS states, and strings Nuclear Phys. B 463 (1996), no. 2-3, $315-368$

[KP] Kac, V.G. and Peterson, D.H. Defining relations of certain infinite dimensional groups, The mathematical heritage of Élie Cartan (Lyon, 1984). Astérisque (1985), Numero Hors Serie, 165-208.

[LT] Lim, S. and Thomas, A. Covering theory for complexes of groups, J. Pure Appl. Algebra, Vol 212, no. 7, (2008), 1632-1663.

[L] Lubotzky, A. Lattices of minimial covolume in $S L_{2}$, Journal of the Amer. Math. Society, 78 (1999), 961-975.

[LW] Lubotzky, A. and Weigel, T. Lattices of minimal covolume in $S L_{2}$ over local fields, Proc. London Math. Soc. 78 (1999), 283-333.

[M] Magnus, W. Residually finite groups, Bull. Amer. Math. Soc. Volume 75, Number 2 (1969), 305-316.

[R] Rosenberg, G. Towers and covolumes of tree lattices, PhD Thesis, Columbia University, (2001).

[Re] Rémy, B. Groupes de Kac-Moody déployés et presque déployés. (French) [Split and almost split Kac-Moody groups] Astérisque No. 277 (2002), viii+348 pp.

[RR] Rémy, B. and Ronan, M. Topological groups of Kac-Moody type, right-angled twinnings and their lattices. Commentarii Mathematici Helvetici 81 (2006), 191-219.

[S] Serre, J.-P. Trees, Translated from the French by John Stillwell. Springer-Verlag, Berlin-New York, (1980). ix +142 pp.

[Sa] Saçliog̃lu, C. Dynkin diagrams for hyperbolic Kac-Moody algebras, Journal of Physics A: Mathematics and General 22, (1989) 3753-3769.

[Ti1] Tits, J. Uniqueness and presentation of Kac-Moody groups over fields Journal of Algebra, 105 (1987), $542-573$.

[Ti2] Tits, J. Resume de Cours - Theorie des Groupes, Annuaire du College de France, 1980-1981, 75-87.

Department of Mathematics, Hill Center, Busch Campus, Rutgers, The State University of New Jersey, 110 Frelinghuysen Rd, Piscataway, NJ 08854-8019

E-mail address: carbonel@math.rutgers.edu

Department of Mathematics, Hill Center, Busch Campus, Rutgers, The State University of New Jersey, 110 Frelinghuysen Rd, Piscataway, NJ 08854-8019

E-mail address: cobbs@math.rutgers.edu 